Research Article

\title{
Identification and Analysis of Chemical Constituents and Rat Serum Metabolites in Gushuling Using UPLC-Q-TOF/MS Coupled with Novel Informatics UNIFI Platform
}

\author{
Hong Chang, ${ }^{1}$ Shujie Lv, ${ }^{1}$ Tengteng Yuan, ${ }^{1}$ Huan $W u,{ }^{2}$ Lei Wang $\mathbb{D},{ }^{1,3}$ Ran Sang $\mathbb{D},{ }^{4,5}$ \\ Caiyun Zhang $\left(\mathbb{0},{ }^{1}\right.$ and Weidong Chen $\mathbb{1}^{1,3}$ \\ ${ }^{1}$ College of Pharmacy, Anhui University of Chinese Medicine, Hefei 230012, China \\ ${ }^{2}$ The Experiment Research Center, Anhui University of Chinese Medicine, Hefei 230012, China \\ ${ }^{3}$ Anhui Province Key Laboratory of Chinese Medicinal Formula, Hefei 230012, China \\ ${ }^{4}$ Bengbu Medical College, Bengbu 233030, China \\ ${ }^{5}$ The First Affiliated Hospital of Bengbu Medical College, Bengbu 233004, China
}

Correspondence should be addressed to Ran Sang; sangzhou1980@126.com, Caiyun Zhang; cyzhang@ahtcm.edu.cn, and Weidong Chen; wdchen@ahtcm.edu.cn

Received 1 October 2021; Accepted 18 November 2021; Published 31 December 2021

Academic Editor: Nianping Feng

Copyright (C) 2021 Hong Chang et al. This is an open access article distributed under the Creative Commons Attribution License, which permits unrestricted use, distribution, and reproduction in any medium, provided the original work is properly cited.

\begin{abstract}
Gushuling (GSL), a well-known hospital preparation composed of traditional Chinese medicine (TCM), has been widely used in the clinical treatment of osteoporosis (OP) for decades due to its remarkable therapeutic effect. However, the chemical constituents of GSL are still unclear so far, which limits the in-depth study of its pharmacodynamic material basis and further restricts its clinical application. In this study, we developed a strategy for qualitative analysis of the chemical constituents of GSL in vitro and in vivo. Based on the results of ultra-performance liquid chromatography coupled with quadrupole time-of-flight tandem mass spectrometry (UPLC-Q-TOF-MS) and the UNIFI informatics platform, the chemical constituents of GSL can be determined quickly and effectively. By comparing the retention time, accurate mass, and fragmentation spectrum of the compounds in GSL, a total of 93 compounds were identified or preliminarily identified, including flavonoids, terpenoids, phenylpropanoids, steroids, etc. Among them, nine compounds have been confirmed by standard substances, namely epimedin A, epimedin B, epimedin C, icariin, ecdysterone, calycosin, calycosin-7-glucoside, ononin, and ginsenoside Ro. Fragment patterns and characteristic ions of representative compounds with different chemical structure types were analyzed. At the same time, 20 prototype compounds and 42 metabolites were detected in rat serum. Oxidation, hydration, reduction, dehydration, glutathione S-conjugation, and acetylcysteine conjugation were the main transformation reactions of GSL in rat serum. In this research, the rapid method to characterize the in vitro and in vivo chemical constituents of GSL can not only be used for the standardization and quality control of GSL but also be helpful for further research on its pharmacodynamic material basis.
\end{abstract}

\section{Introduction}

Osteoporosis (OP) is a complex bone disease characterized by low bone density and impaired microstructure of bone tissue, leading to increased bone fragility and being prone to fractures [1]. At present, the treatment drugs for OP mainly include ingredients that inhibit bone resorption, such as bisphosphonates, calcitonin, and estrogen, and ingredients that promote bone formation, such as parathyroid hormone
[2]. However, taking bisphosphonates for a long time will increase the incidence of complications such as osteonecrosis of the jaw $[3,4]$. Long-term use of estrogen will also increase the incidence of breast cancer, endometrial cancer, and other diseases [5,6]. Nowadays, the importance of traditional Chinese medicine (TCM) and Chinese herbal compound prescription that have a therapeutic effect on OP were gradually been recognized because of their safety and effectiveness [7-9]. 
Gushuling (GSL) is a TCM preparation in the hospital of the First Affiliated Hospital of Anhui University of Chinese Medicine, consisting of Herba Epimedii (Yinyanghuo), Radix Achyranthis Bidentatae (Niuxi), Radix Astragali (Huangqi), and Concha Ostreae (Muli) as recorded in the Chinese Pharmacopoeia (Table S1). GSL has a good effect on the management of various types of OP in clinical treatment, such as senile osteoporosis [10,11], postmenopausal osteoporosis [12], and diabetes secondary osteoporosis [13]. However, the chemical constituents of TCM and related preparations are complex and the active ingredients are not fully understood $[14,15]$. Although the chemical constituents of GSL have been previously reported, only two or three bioactive ingredients have been found in GSL [16,17]. These efforts failed to reflect the overall chemical constituents of GSL, which made it was difficult to comprehensively evaluate the quality of GSL. Therefore, there is an urgent need for a reliable and efficient analytical method to determine the chemical constituents of GSL and the effective ingredients that can enter the body to achieve the purpose of quality control. Fortunately, the combination of ultra-high-performance liquid chromatography coupled with quadrupole time-of-flight tandem mass spectrometry (UPLC-Q-TOFMS) and the UINIF analysis platform can solve this problem to some extent.

In recent years, UPLC-Q-TOF-MS has been widely used in various fields such as qualitative analysis of TCM ingredients, serum pharmacochemistry, metabolomics, and new drug development due to its high resolution and high sensitivity [18-21]. As a powerful information processing platform, UNIFI can automatically extract the mass spectrometry data of the sample, determine the molecular formula, compare it with the compound in the database, which gives the possible cracking method of the compound according to the fragment information of the compound under high energy, and display the detailed information of the identified compound under the preset filter conditions $[22,23]$. The purpose of this study was to use the UPLC-QTOF-MS technology in conjunction with UNIFI analysis software to comprehensively analyze and identify the chemical constituents of hospital preparation GSL and to analyze the prototype compounds and metabolites in rat serum, which provides a basis for the in-depth research and quality control of its pharmacodynamic material basis.

\section{Materials and Methods}

2.1. Materials and Reagents. Herba Epimedii, Radix Achyranthis Bidentatae, Radix Astragali, and Concha Ostreae were purchased from Anhui Xiehecheng Co., Ltd. (Bozhou, China) and evaluated by Doctor Rongchun Han (College of Pharmacy, Anhui University of Chinese Medicine, Hefei, China). The standard substances of calycosin (Lot number: MUST-18120901), calycosin-7-glucoside (Lot number: MUST-19031920), ononin (Lot number: MUST-19041101), epimedin C (Lot number: MUST-19081310), icariin (Lot number: MUST-18091010), and ecdysterone (Lot number: MUST-18120209) were purchased from Chengdu Must BioTechnology Co., Ltd. (Chengdu, China). Epimedin A (Lot number: JOT-10354), epimedin B (Lot number: JOT-10353), and ginsenoside Ro (Lot number: JOT-10448) were purchased from Chengdu Pufei De Biotech Co., Ltd. (Chengdu, China). The purity of all standards was over 98.0\%. Acetonitrile (HPLC grade; Lot number: SHBM1775) and methanol were purchased from Sigma Aldrich Trading Co., Ltd. (Shanghai, China). Formic acid (HPLC grade; Lot number: J2027129) was obtained from Aladdin Biochemical Technology Co., Ltd. (Shanghai, China). Ultrapure water was prepared by a Milli Q-Plus system (Millipore, Bedford, USA).

2.2. Standards and Sample Preparation. First, Herba Epimedii and Radix Achyranthis Bidentatae were mixed at the ratio of $5: 4$ and refluxed with $70 \%$ ethanol at 8 -fold volume (11-fold volume for the first time) for 3 times for $2 \mathrm{~h}$, respectively. We used a rotary evaporator to volatilize the ethanol in the extract to obtain a concentrated solution for use. Second, Radix Astragali and Concha Ostreae were mixed at the ratio of 5:2 and boiled with 9.5-fold and 8-fold volume ultrapure water for $1 \mathrm{~h}$, respectively. Finally, the ethanol extract obtained in the first step was mixed with the water extract obtained in the second step and concentrated to obtain the GSL sample solution. Herba Epimedii refluxed with $70 \%$ ethanol at 8 -fold volume (11-fold volume for the first time) for 3 times for $2 \mathrm{~h}$, respectively. We used a rotary evaporator to volatilize the ethanol and water in the extract to obtain a sample solution of Herba Epimedii. The preparation method of Radix Achyranthis Bidentatae sample solution is the same as that of Herba Epimedii sample solution. Radix Astragali boiled with 9.5-fold and 8-fold volume ultrapure water for $1 \mathrm{~h}$, respectively, and concentrated to obtain the Radix Astragali sample solution. An appropriate amount of sample solution was diluted with methanol, and the supernatant was taken and stored at $4^{\circ} \mathrm{C}$. All the solutions were filtered through a $0.22 \mu \mathrm{m}$ filter membrane before analysis.

Nine standard substances were completely dissolved in methanol. Before qualitative analysis, they were mixed to prepare a mixed standard solution with appropriate concentration and passed through a $0.22 \mu \mathrm{m}$ filter membrane. All solutions were stored in a refrigerator at $4^{\circ} \mathrm{C}$.

\subsection{Animals Handling and Serum Samples Preparation.} Sprague Dawley rats $(200 \pm 20 \mathrm{~g})$ of specific pathogen-free grade were purchased from Animal Experiment Center, Bengbu Medical College. Approved by the Experimental Animal Management and Ethics Committee of Bengbu Medical College, animal experimental research meets relevant ethical requirements. They were randomly divided into blank groups and GSL extract groups, with 6 rats in each group. The rats were kept in an animal room with a suitable environment for 7 days before the experiment. Rats in the blank group and GSL extract group were intragastrically administered distilled water and $51.993 \mathrm{~g} \cdot \mathrm{kg}^{-1} \cdot \mathrm{d}^{-1} \mathrm{GSL}$ sample solution for consecutive 3 days, respectively. The preparation process of the GSL sample solution is the same as that in the Standards and Sample Preparation section, and 
there is no need for subsequent operations such as methanol treatment. Before the last oral administration, the rats fasted for $12 \mathrm{~h}$ with free drinking water. Blood samples $(500 \mu \mathrm{L})$ were collected from the fundus venous plexus $15 \mathrm{~min}$ after oral administration on the 3rd day and centrifuged for $10 \mathrm{~min}$ at $3500 \mathrm{rpm} \cdot \mathrm{min}^{-1}$ at $4^{\circ} \mathrm{C}$, and the supernatant was taken. Then, $1200 \mu \mathrm{L}$ methanol was added to the $300 \mu \mathrm{L}$ serum samples, vortexed, and centrifuged at $13000 \mathrm{rpm}$ for $10 \mathrm{~min}$. The supernatant was put into another tube and dried with nitrogen gas. The remaining was stored in acetonitrile $(200 \mu \mathrm{L})$ and frozen at $-80^{\circ} \mathrm{C}$ until analysis.

2.4. Chromatography and Mass Spectrometry Conditions. Chromatographic analysis was performed using a Waters Acquity $^{\mathrm{TM}}$ UPLC system (Waters Corporation, Milford, USA). Chromatographic separation was carried on Waters ACQUITY UPLC ${ }^{\circledR}$ BEH C18 $(2.1 \times 100 \mathrm{~mm}, 1.7 \mu \mathrm{m})$ column by gradient elution with the optimal mobile phase of $0.1 \%$ formic acid aqueous solution (solvent A) and acetonitrile (solvent B), the column temperature was maintained at $35^{\circ} \mathrm{C}$, and the temperature of the sample chamber was set to $8^{\circ} \mathrm{C}$. The gradient elution was set as follows: $0-5 \mathrm{~min}, 5 \%-19 \% \mathrm{~B}$; 5-10 min, $19 \% \mathrm{~B} ; 10-11 \mathrm{~min}, 19 \%-25 \% \mathrm{~B} ; 11-16 \mathrm{~min}, 25 \%$ B; $16-17 \mathrm{~min}, \quad 25 \%-31 \% \mathrm{~B} ; 17-22 \mathrm{~min}, 31 \%-51 \% \quad \mathrm{~B}$; $22-50 \mathrm{~min}, 51 \%-100 \% \mathrm{~B}$; $50-55 \mathrm{~min}, 100 \% \mathrm{~B}$; $55-60 \mathrm{~min}$, $100 \%-5 \% \mathrm{~B} ; 60-65 \mathrm{~min}, 5 \% \mathrm{~B}$. The flow rate was $0.15 \mathrm{~mL} \cdot \mathrm{min}^{-1}$, and the injection volume was $2 \mu \mathrm{L}$.

A Waters Xevo G2 Q-TOF mass spectrometer (Waters Corporation, Milford, USA) equipped with an electrospray ionization (ESI) source operating in both positive and negative ion modes was connected to the UPLC. The full scan data were collected from $\mathrm{m} / \mathrm{z} 50$ to $\mathrm{m} / \mathrm{z} 1200$. For positive and negative ion modes, the capillary and cone voltage were set to $3.0 \mathrm{kV}, 40 \mathrm{~V}$ and $2.5 \mathrm{kV}, 40 \mathrm{~V}$, respectively. The temperature of the conservation gas was set to $350^{\circ} \mathrm{C}$, and the flow rate was set to $600 \mathrm{~L} \cdot \mathrm{h}^{-1}$. The two ion source temperatures in positive ion and negative ion modes were set to $120^{\circ} \mathrm{C}$ and $110^{\circ} \mathrm{C}$, respectively. The cone gas flow rate was set to $50 \mathrm{~L} \cdot \mathrm{h}^{-1}$, and leucine and enkephalin were used as calibration fluids to ensure accuracy and repeatability.

2.5. UNIFI Data Processing Method. The chemical constituents analysis strategy was mainly divided into the following three steps:

(1) The establishment of the chemical constituents library of GSL: The complete information on the compounds of three herbal medicines (Herba Epimedii, Radix Achyranthis Bidentatae, and Radix Astragali) in GSL was collected and obtained by searching China National Knowledge Infrastructure (CNKI), PubMed, PubChem, Traditional Chinese Medicine Systems Pharmacology Database and Analysis Platform (TCMSP), ChemSpider, and other databases. The self-built compound library was established, including compound name, molecular formula, chemical structure (saved in "mol" format), accurate molecular mass, and other information. This information was imported into UNIFI. Among them, a total of 354 compounds were listed. When analyzing rat serum samples, the self-built database imported into UNIFI was the GSL in vitro compound information database that has been analyzed and confirmed.

(2) Preliminary analysis of the results: We imported the original files of GSL sample solution, blank sample solution, rat administration serum, and blank serum analyzed by UPLC-Q-TOF-MS into the UNIFI software for samples comparison. Based on the automatic matching function of UNIFI software, compounds can be quickly identified. The parameter settings were as follows: analysis time range, $1-65 \mathrm{~min}$; quality allowable error range, $\pm 10 \mathrm{ppm}$; quality testing range, $50 \mathrm{Da}$ to $1500 \mathrm{Da}$; positive adducts including $\mathrm{H}^{+}, \mathrm{Na}^{+}$, and $\mathrm{K}^{+}$; and negative adducts containing $\mathrm{H}^{-}, \mathrm{HCOO}^{-}$, and $\mathrm{Cl}^{-}$.

(3) Manual review: By the MassLynx workstation, the above identification results were reviewed in combination with the precise mass of excimer ions, retention time, fragment ion information, and literature.

\section{Results and Discussion}

3.1. Chemical Constituents Analysis of Three Herbal Medicines in GSL. The separate extraction method of the three herbal medicines in GSL (Herba Epimedii, Radix Achyranthis Bidentatae, and Radix Astragali) was the same as the sample preparation process. Then, the TIC of the three herbal medicines in positive and negative ion modes were qualitatively analyzed according to the above-mentioned method, and the results are shown in Figure S1.

\subsection{Identification and Analysis of Chemical Constituents in} GSL. High-resolution mass spectrometry data of GSL were rapidly acquired by the UPLC-Q-TOF-MS method. The TIC of GSL in positive and negative ion modes is portrayed in Figure 1. The UNIFI screening platform was used to process and analyze its mass spectrometry data, and then the data were spontaneously matched with the fragment information. After further manual verification, it was found that there were 93 in vitro chemical constituents in the GSL recipe, including 51 kinds of flavonoids, 18 kinds of terpenoids, 6 kinds of phenylpropanoids, 5 kinds of steroids, and 13 others. Among them, 55 compounds were from Herba Epimedii, 23 compounds from Radix Achyranthis Bidentatae, and 21 compounds from Radix Astragali. In addition, Herba Epimedii and Radix Achyranthis Bidentatae contain four common compounds, and Herba Epimedii, Radix Achyranthis Bidentatae, and Radix Astragali contain one identical compound (rutin). Of the identified components, 9 compounds were identified by comparison with the standard substance. The detailed information on these components is gathered in Table 1 and Figure 2. 

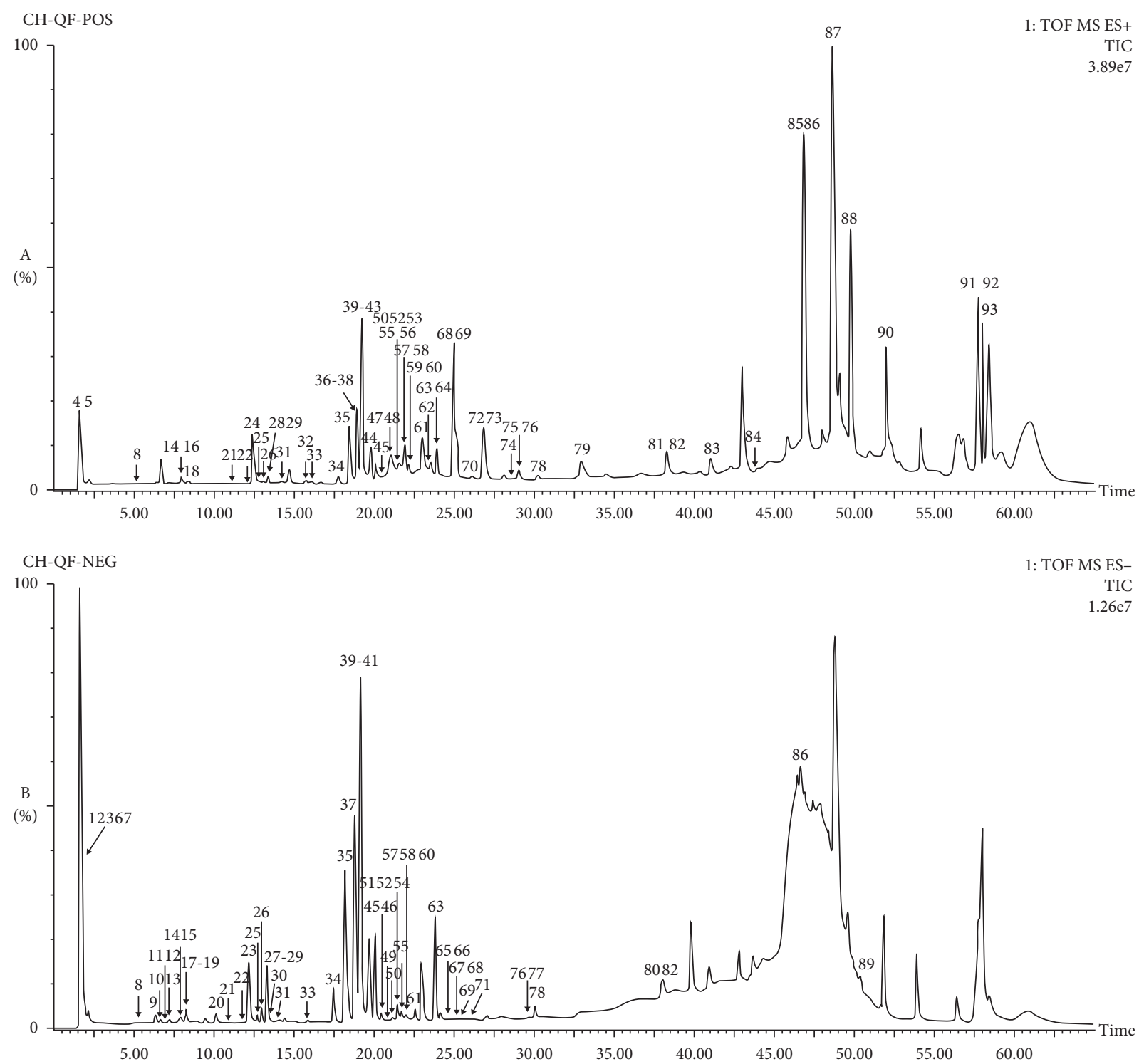

Figure 1: The total ion chromatography (TIC) of GSL from UPLC-Q-TOF-MS analysis. (a) Positive ion mode. (b) Negative ion mode.

TABLE 1: Identification of chemical constituents of GSL by UPLC-Q-TOF-MS.

\begin{tabular}{|c|c|c|c|c|c|c|c|c|c|}
\hline NO. & $\begin{array}{l}\text { Observed } \\
\text { RT (min) }\end{array}$ & Formula & $\begin{array}{c}\text { Observed } \\
\mathrm{m} / \mathrm{z}\end{array}$ & $\begin{array}{l}\text { Mass } \\
\text { error } \\
(\mathrm{ppm})\end{array}$ & Adducts & $\begin{array}{c}\text { Fragment } \\
(+/-)\end{array}$ & Identification & Structure class & Origin \\
\hline 1 & 1.63 & $\mathrm{C}_{6} \mathrm{H}_{12} \mathrm{O}_{5}$ & 209.0670 & 1.3 & {$[\mathrm{M}+\mathrm{HCOO}]^{-}$} & 89.0255 & RAM & Saccharide & HQ \\
\hline 2 & 1.64 & $\mathrm{C}_{5} \mathrm{H}_{10} \mathrm{O}_{5}$ & 195.0510 & -0.2 & {$[\mathrm{M}+\mathrm{HCOO}]^{-}$} & 89.0255 & XLS & Saccharide & HQ \\
\hline 3 & 1.66 & $\mathrm{C}_{23} \mathrm{H}_{24} \mathrm{O}_{11}$ & 475.1287 & 8.6 & {$[\mathrm{M}-\mathrm{H}]^{-}$} & $\begin{array}{c}89.0255 \\
179.0563 \\
290.0864 \\
341.1084\end{array}$ & $\begin{array}{c}7,2^{\prime} \text {-Dihydroxy- } 3^{\prime}, 4^{\prime}- \\
\text { dimethoxyisoflavone-7- } \\
\text { O- } \beta \text {-D-glucoside }\end{array}$ & Flavonoids & HQ \\
\hline 4 & 1.67 & $\mathrm{C}_{6} \mathrm{H}_{12} \mathrm{O}_{6}$ & 203.0522 & -2.2 & $\begin{array}{c}{[\mathrm{M}+\mathrm{Na}]^{+},} \\
{[\mathrm{M}+\mathrm{K}]^{+}}\end{array}$ & 127.0388 & Inositol & Others & YYH \\
\hline 5 & 1.68 & $\mathrm{C}_{10} \mathrm{H}_{14} \mathrm{O}_{3}$ & 205.0836 & 0.4 & {$[\mathrm{M}+\mathrm{Na}]^{+}$} & $\begin{array}{l}127.0388 \\
89.0255\end{array}$ & 3,4,5-Trimethoxytoluene & Others & YYH \\
\hline 6 & 1.69 & $\mathrm{C}_{27} \mathrm{H}_{32} \mathrm{O}_{12}$ & 593.1879 & 0.6 & {$[\mathrm{M}+\mathrm{HCOO}]^{-}$} & $\begin{array}{l}179.0563 \\
290.0864 \\
341.1084 \\
377.0850\end{array}$ & Maohuoside A & Flavonoids & YYH \\
\hline 7 & 1.76 & $\mathrm{C}_{21} \mathrm{H}_{22} \mathrm{O}_{7}$ & 431.1381 & 7.8 & {$[\mathrm{M}+\mathrm{HCOO}]^{-}$} & 128.0362 & Wanepimedoside_qt & Flavonoids & YYH \\
\hline
\end{tabular}


TABle 1: Continued.

\begin{tabular}{|c|c|c|c|c|c|c|c|c|c|}
\hline NO. & $\begin{array}{l}\text { Observed } \\
\text { RT (min) }\end{array}$ & Formula & $\begin{array}{l}\text { Observed } \\
\mathrm{m} / \mathrm{z}\end{array}$ & $\begin{array}{l}\text { Mass } \\
\text { error } \\
(\mathrm{ppm})\end{array}$ & Adducts & $\begin{array}{l}\text { Fragment } \\
\qquad(+/-)\end{array}$ & Identification & Structure class & Origin \\
\hline \multirow[t]{2}{*}{8} & 5.25 & $\mathrm{C}_{16} \mathrm{H}_{18} \mathrm{O}_{9}$ & 353.0867 & -3.1 & {$[\mathrm{M}-\mathrm{H}]^{-}$} & 191.0556 & Chlorogenic acid & $\begin{array}{l}\text { Phenylpropanoids } \\
\text { (phenylpropionic } \\
\text { acid) }\end{array}$ & YYH \\
\hline & & $\mathrm{C}_{16} \mathrm{H}_{18} \mathrm{O}_{9}$ & 355.1041 & 4.9 & $\begin{array}{l}{[\mathrm{M}+\mathrm{H}]^{+}} \\
{[\mathrm{M}+\mathrm{Na}]^{+}}\end{array}$ & 163.0387 & Chlorogenic acid & $\begin{array}{l}\text { Phenylpropanoids } \\
\text { (phenylpropionic } \\
\text { acid) }\end{array}$ & YYH \\
\hline 9 & 6.44 & $\mathrm{C}_{19} \mathrm{H}_{30} \mathrm{O}_{8}$ & 431.189 & -6.6 & {$[\mathrm{M}+\mathrm{HCOO}]^{-}$} & $\begin{array}{l}163.0409 \\
173.0463 \\
219.0629\end{array}$ & Icariside $\mathrm{B} 1$ & $\begin{array}{c}\text { Terpenoids } \\
\text { (monocyclic } \\
\text { monoterpenoids) }\end{array}$ & YYH \\
\hline 10 & 6.49 & $\mathrm{C}_{19} \mathrm{H}_{28} \mathrm{O}_{10}$ & 461.1704 & 8.6 & {$[\mathrm{M}+\mathrm{HCOO}]^{-}$} & 219.0629 & Icariside D1 & $\begin{array}{c}\text { Terpenoids } \\
\text { (monocyclic } \\
\text { monoterpenoids) }\end{array}$ & YYH \\
\hline 11 & 6.87 & $\mathrm{C}_{26} \mathrm{H}_{36} \mathrm{O}_{12}$ & 539.2149 & 2.7 & {$[\mathrm{M}-\mathrm{H}]^{-}$} & $\begin{array}{l}317.0238 \\
479.0789 \\
491.1857\end{array}$ & Icariside E1 & $\begin{array}{l}\text { Phenylpropanoids } \\
\text { (phenylpropanols) }\end{array}$ & YYH \\
\hline 12 & 6.92 & $\mathrm{C}_{21} \mathrm{H}_{20} \mathrm{O}_{13}$ & 479.0807 & -5.1 & {$[\mathrm{M}-\mathrm{H}]^{-}$} & $\begin{array}{l}316.0208 \\
191.0563\end{array}$ & Isomyricitrin & Flavonoids & YYH \\
\hline 13 & 7.22 & $\mathrm{C}_{32} \mathrm{H}_{38} \mathrm{O}_{15}$ & 697.1844 & -8.8 & {$[\mathrm{M}+\mathrm{Cl}]^{-}$} & $\begin{array}{r}219.0647 \\
593.1515\end{array}$ & Ikarisoside B & Flavonoids & YYH \\
\hline 14 & 7.86 & $\begin{array}{l}\mathrm{C}_{22} \mathrm{H}_{22} \mathrm{O}_{10} \\
\mathrm{C}_{22} \mathrm{H}_{22} \mathrm{O}_{10}\end{array}$ & $\begin{array}{l}491.1183 \\
447.1279\end{array}$ & $\begin{array}{l}-2.5 \\
-1.4\end{array}$ & $\frac{[\mathrm{M}+\mathrm{HCOO}]^{-}}{[\mathrm{M}+\mathrm{H}]^{+}}$ & $\begin{array}{l}283.0601 \\
285.0749\end{array}$ & $\begin{array}{l}\text { Calycosin-7-glucoside* } \\
\text { Calycosin-7-glucoside* }\end{array}$ & $\begin{array}{l}\text { Flavonoids } \\
\text { Flavonoids }\end{array}$ & $\begin{array}{l}\text { HQ } \\
\text { HQ }\end{array}$ \\
\hline 15 & 7.92 & $\mathrm{C}_{21} \mathrm{H}_{20} \mathrm{O}_{12}$ & 463.0849 & -7.1 & {$[\mathrm{M}-\mathrm{H}]^{-}$} & $\begin{array}{l}\text { 285.0396, } \\
431.0962\end{array}$ & Hyperoside & Flavonoids & $\begin{array}{l}\text { YYH } \\
\text { NX }\end{array}$ \\
\hline 16 & 8.00 & $\mathrm{C}_{15} \mathrm{H}_{10} \mathrm{O}_{7}$ & 303.0520 & 6.8 & {$[\mathrm{M}+\mathrm{H}]^{+}$} & $\begin{array}{l}287.0543 \\
193.0869\end{array}$ & Robinetin & Flavonoids & YYH \\
\hline 17 & 8.17 & $\mathrm{C}_{28} \mathrm{H}_{34} \mathrm{O}_{12}$ & 597.1798 & 9.1 & {$[\mathrm{M}+\mathrm{Cl}]^{-}$} & $\begin{array}{l}219.0659 \\
237.0750 \\
399.1276\end{array}$ & Caohuoside D & Flavonoids & YYH \\
\hline \multirow[t]{2}{*}{18} & 8.39 & $\mathrm{C}_{27} \mathrm{H}_{44} \mathrm{O}_{7}$ & 525.3065 & -0.8 & $\begin{array}{c}{[\mathrm{M}+\mathrm{HCOO}]^{-}} \\
{[\mathrm{M}+\mathrm{Cl}]^{-}}\end{array}$ & $\begin{array}{l}159.1057 \\
193.0869 \\
219.0659\end{array}$ & Ecdysterone* & Steroids & NX \\
\hline & & $\mathrm{C}_{27} \mathrm{H}_{44} \mathrm{O}_{7}$ & 481.3134 & -5.5 & $\begin{array}{l}{[\mathrm{M}+\mathrm{K}]^{+}} \\
{[\mathrm{M}+\mathrm{Na}]^{+}}\end{array}$ & $\begin{array}{l}371.2244 \\
445.2923\end{array}$ & Ecdysterone* & Steroids & NX \\
\hline 19 & 8.42 & $\mathrm{C}_{22} \mathrm{H}_{32} \mathrm{O}_{11}$ & 517.1889 & -7.2 & $\begin{array}{c}{[\mathrm{M}+\mathrm{HCOO}]^{-},} \\
{[\mathrm{M}-\mathrm{H}]^{-},} \\
{[\mathrm{M}+\mathrm{Cl}]^{-}}\end{array}$ & $\begin{array}{l}177.0907 \\
471.1833\end{array}$ & Eugenol rutinoside & $\begin{array}{l}\text { Phenylpropanoids } \\
\text { (Styrene) }\end{array}$ & YYH \\
\hline 20 & 10.13 & $\mathrm{C}_{29} \mathrm{H}_{38} \mathrm{O}_{15}$ & 625.2131 & -1.1 & {$[\mathrm{M}-\mathrm{H}]^{-}$} & $\begin{array}{l}261.0762, \\
279.0868, \\
291.0852\end{array}$ & $\begin{array}{c}\text { Isomucronulatol-7,2' } \text {-di- } \\
\text { O-glucosiole }\end{array}$ & Flavonoids & HQ \\
\hline 21 & 11.14 & $\mathrm{C}_{16} \mathrm{H}_{12} \mathrm{O}_{5}$ & 283.0599 & -4.7 & {$[\mathrm{M}-\mathrm{H}]^{-}$} & 268.0381 & Wogonin & Flavonoids & NX \\
\hline \multirow{3}{*}{22} & & $\mathrm{C}_{16} \mathrm{H}_{12} \mathrm{O}_{5}$ & 285.0769 & 4.0 & {$[\mathrm{M}+\mathrm{H}]^{+}$} & $\begin{array}{l}270.0515 \\
353.0993\end{array}$ & Wogonin & Flavonoids & NX \\
\hline & 12.07 & $\mathrm{C}_{32} \mathrm{H}_{38} \mathrm{O}_{16}$ & 677.2074 & -1.9 & {$[\mathrm{M}-\mathrm{H}]^{-}$} & $\begin{array}{l}514.1457 \\
515.1522 \\
557.1635\end{array}$ & Hexandraside E & Flavonoids & YYH \\
\hline & & $\mathrm{C}_{32} \mathrm{H}_{38} \mathrm{O}_{16}$ & 679.2231 & -0.3 & $\begin{array}{l}{[\mathrm{M}+\mathrm{H}]^{+}} \\
{[\mathrm{M}+\mathrm{Na}]^{+}}\end{array}$ & $\begin{array}{l}299.0524 \\
355.1186 \\
517.1787\end{array}$ & Hexandraside E & Flavonoids & YYH \\
\hline 23 & 12.18 & $\mathrm{C}_{43} \mathrm{H}_{68} \mathrm{O}_{14}$ & 853.4619 & 3.3 & $\begin{array}{l}{[\mathrm{M}+\mathrm{HCOO}]^{-}} \\
{[\mathrm{M}-\mathrm{H}]^{-}}\end{array}$ & $\begin{array}{l}96.9603 \\
451.3268\end{array}$ & $\begin{array}{c}\text { 1-O- }\{(3 \beta, 5 \xi, 9 \xi)-3-[(6- \\
\text { Methyl- } \beta \text {-D- } \\
\text { glucopyranuronosyl) } \\
\text { oxy]-28-oxoolean-12- } \\
\text { en-28-yl }\}-\beta-\mathrm{D}- \\
\text { glucopyranose }\end{array}$ & Triterpenoids & NX \\
\hline 24 & 12.41 & $\mathrm{C}_{29} \mathrm{H}_{50} \mathrm{O}$ & 453.3471 & -4.8 & {$[\mathrm{M}+\mathrm{K}]^{+}$} & 343.3055 & Sitosterol & Steroids & $\begin{array}{l}\text { YYH } \\
\text { NX }\end{array}$ \\
\hline
\end{tabular}


TABle 1: Continued.

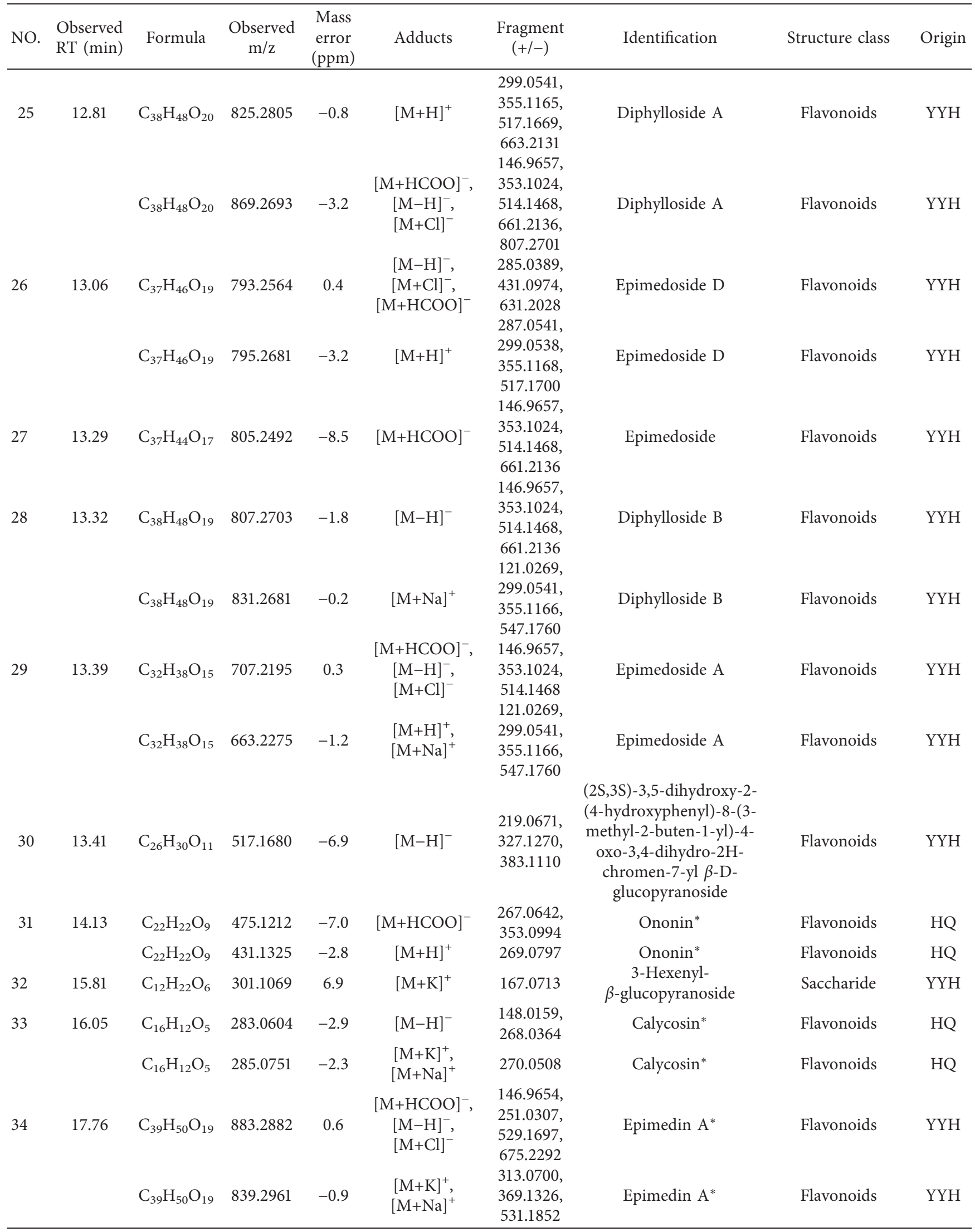


TABle 1: Continued.

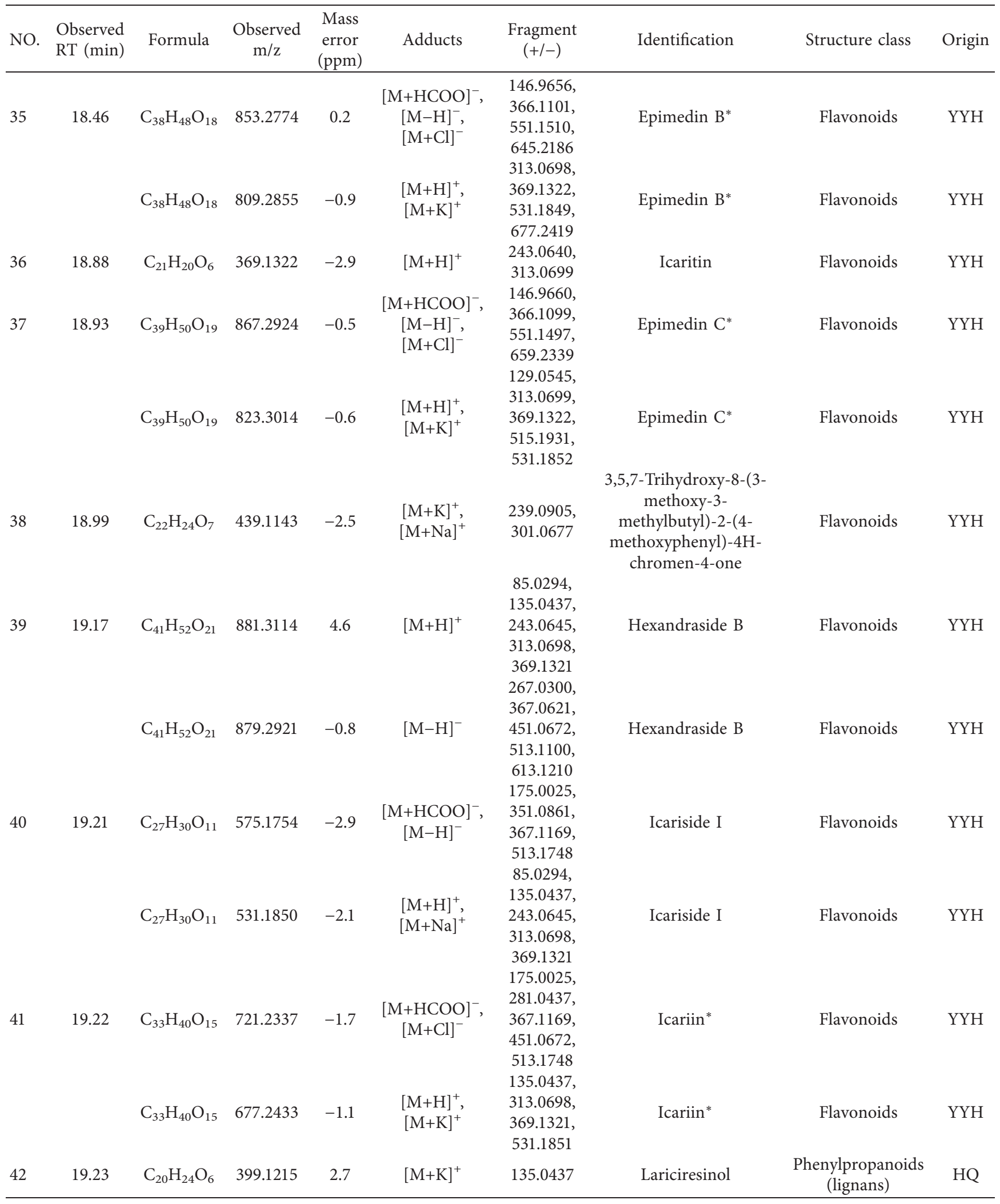


TABle 1: Continued.

\begin{tabular}{|c|c|c|c|c|c|c|c|c|c|}
\hline NO. & $\begin{array}{l}\text { Observed } \\
\text { RT (min) }\end{array}$ & Formula & $\begin{array}{l}\text { Observed } \\
\mathrm{m} / \mathrm{z}\end{array}$ & $\begin{array}{l}\text { Mass } \\
\text { error } \\
(\mathrm{ppm}) \\
\end{array}$ & Adducts & $\begin{array}{l}\text { Fragment } \\
(+/-)\end{array}$ & Identification & Structure class & Origin \\
\hline 43 & 19.26 & $\mathrm{C}_{28} \mathrm{H}_{24} \mathrm{O}_{13}$ & 569.1236 & -9.4 & {$[\mathrm{M}+\mathrm{H}]^{+}$} & $\begin{array}{c}85.0294 \\
135.0437 \\
313.0698 \\
369.1321\end{array}$ & $\begin{array}{c}\text { 8-Hydroxy-6-methyl- } \\
\text { 9,10-dioxo-9,10- } \\
\text { dihydro-1-anthracenyl } \\
\text { 6-O- }(3,4,5- \\
\text { trihydroxybenzoyl)- } \beta \text {-D- } \\
\text { glucopyranoside }\end{array}$ & Anthraquinones & NX \\
\hline 44 & 19.63 & $\mathrm{C}_{25} \mathrm{H}_{30} \mathrm{O}_{8}$ & 481.1785 & -9.9 & {$[\mathrm{M}+\mathrm{Na}]^{+}$} & 387.1427 & Rubschisantherin & $\begin{array}{c}\text { Phenylpropanoids } \\
\text { (lignans) }\end{array}$ & NX \\
\hline \multirow[t]{2}{*}{45} & 20.82 & $\mathrm{C}_{36} \mathrm{H}_{42} \mathrm{O}_{17}$ & 747.2483 & -1.5 & {$[\mathrm{M}+\mathrm{H}]^{+}$} & $\begin{array}{l}299.0532 \\
355.1114\end{array}$ & Korepimedoside A & Flavonoids & YYH \\
\hline & & $\mathrm{C}_{36} \mathrm{H}_{42} \mathrm{O}_{17}$ & 745.2332 & -2.3 & {$[\mathrm{M}-\mathrm{H}]^{-}$} & $\begin{array}{c}121.0304 \\
352.0934 \\
367.1168 \\
499.1620 \\
583.1812\end{array}$ & Korepimedoside A & Flavonoids & YYH \\
\hline 46 & 20.94 & $\mathrm{C}_{41} \mathrm{H}_{52} \mathrm{O}_{21}$ & 879.2892 & -4.1 & {$[\mathrm{M}-\mathrm{H}]^{-}$} & $\begin{array}{l}367.1156 \\
381.0964 \\
571.1715 \\
673.2076\end{array}$ & Korepimedoside C & Flavonoids & YYH \\
\hline 47 & 21.01 & $\mathrm{C}_{56} \mathrm{H}_{88} \mathrm{O}_{25}$ & 1199.5264 & 1.5 & {$[\mathrm{M}+\mathrm{K}]^{+}$} & $\begin{array}{c}369.1320 \\
383.1110 \\
882.4549 \\
1059.5290\end{array}$ & $\begin{array}{l}\text { Achyranthoside } D \\
\text { trimethyl ester }\end{array}$ & Triterpenoids & NX \\
\hline 48 & 21.08 & $\mathrm{C}_{52} \mathrm{H}_{84} \mathrm{O}_{19}$ & 1013.5723 & 4.3 & {$[\mathrm{M}+\mathrm{H}]^{+}$} & $\begin{array}{l}295.0576 \\
311.0891 \\
385.1281 \\
882.4549\end{array}$ & $\begin{array}{c}\text { Chikusetsusaponin V } \\
\text { butyl ester }\end{array}$ & Triterpenoids & NX \\
\hline 49 & 21.09 & $\mathrm{C}_{53} \mathrm{H}_{82} \mathrm{O}_{25}$ & 1117.5026 & -4.1 & {$[\mathrm{M}-\mathrm{H}]^{-}$} & $\begin{array}{l}312.0607 \\
383.1097 \\
529.1652 \\
631.2021 \\
997.4956\end{array}$ & Achyranthoside D & Triterpenoids & NX \\
\hline \multirow[t]{2}{*}{50} & 21.11 & $\mathrm{C}_{47} \mathrm{H}_{74} \mathrm{O}_{18}$ & 949.4763 & -0.5 & {$[\mathrm{M}+\mathrm{Na}]^{+}$} & $\begin{array}{l}295.0576 \\
311.0891 \\
882.4549\end{array}$ & Chikusetsusaponin IV & Triterpenoids & NX \\
\hline & & $\mathrm{C}_{47} \mathrm{H}_{74} \mathrm{O}_{18}$ & 925.4757 & -4.9 & {$[\mathrm{M}-\mathrm{H}]^{-}$} & $\begin{array}{l}139.1120 \\
171.1024 \\
211.1335 \\
229.1434 \\
367.1184\end{array}$ & Chikusetsusaponin IV & Triterpenoids & NX \\
\hline 51 & 21.19 & $\mathrm{C}_{48} \mathrm{H}_{76} \mathrm{O}_{19}$ & 955.4884 & -2.5 & $\begin{array}{c}{[\mathrm{M}-\mathrm{H}]^{-}} \\
{[\mathrm{M}+\mathrm{HCOO}]^{+}}\end{array}$ & $\begin{array}{l}352.0934 \\
367.1168 \\
631.2021 \\
793.4236\end{array}$ & Ginsenoside Ro* & Triterpenoids & NX \\
\hline 52 & 21.24 & $\mathrm{C}_{31} \mathrm{H}_{36} \mathrm{O}_{14}$ & 631.2021 & -1.8 & {$[\mathrm{M}-\mathrm{H}]^{-}$} & $\begin{array}{c}121.0304 \\
352.0934 \\
367.1168 \\
583.1812\end{array}$ & Ikarisoside F & Flavonoids & YYH \\
\hline & & $\mathrm{C}_{31} \mathrm{H}_{36} \mathrm{O}_{14}$ & 633.2146 & -5.0 & {$[\mathrm{M}+\mathrm{H}]^{+}$} & $\begin{array}{l}299.0526 \\
369.1327 \\
385.1281\end{array}$ & Ikarisoside $\mathrm{F}$ & Flavonoids & YYH \\
\hline 53 & 21.28 & $\mathrm{C}_{45} \mathrm{H}_{60} \mathrm{O}_{24}$ & 1023.3186 & 7.8 & {$[\mathrm{M}+\mathrm{K}]^{+}$} & $\begin{array}{l}299.0526 \\
369.1327 \\
531.1847 \\
915.3315\end{array}$ & Acuminatoside & Flavonoids & YYH \\
\hline
\end{tabular}


TABle 1: Continued.

\begin{tabular}{|c|c|c|c|c|c|c|c|c|c|}
\hline NO. & $\begin{array}{l}\text { Observed } \\
\text { RT (min) }\end{array}$ & Formula & $\begin{array}{c}\text { Observed } \\
\mathrm{m} / \mathrm{z}\end{array}$ & $\begin{array}{l}\text { Mass } \\
\text { error } \\
(\mathrm{ppm})\end{array}$ & Adducts & $\begin{array}{l}\text { Fragment } \\
\quad(+/-)\end{array}$ & Identification & Structure class & Origin \\
\hline 54 & 21.31 & $\mathrm{C}_{27} \mathrm{H}_{30} \mathrm{O}_{11}$ & 529.1669 & -8.8 & {$[\mathrm{M}-\mathrm{H}]^{-}$} & $\begin{array}{l}121.0304 \\
219.0656 \\
383.1102 \\
513.1729\end{array}$ & Wushanicariin & Flavonoids & YYH \\
\hline \multirow[t]{2}{*}{55} & \multirow[t]{2}{*}{21.56} & $\mathrm{C}_{35} \mathrm{H}_{42} \mathrm{O}_{16}$ & 763.2415 & -5.2 & {$[\mathrm{M}+\mathrm{HCOO}]^{-}$} & $\begin{array}{l}367.1184 \\
381.0975 \\
555.1833 \\
645.2117\end{array}$ & Sagittatoside C & Flavonoids & YYH \\
\hline & & $\mathrm{C}_{35} \mathrm{H}_{42} \mathrm{O}_{16}$ & 719.2591 & 6.3 & {$[\mathrm{M}+\mathrm{H}]^{+}$} & $\begin{array}{c}313.0706 \\
369.1324 \\
383.1134 \\
385.1288 \\
531.1848\end{array}$ & Sagittatoside C & Flavonoids & YYH \\
\hline 56 & 21.61 & $\mathrm{C}_{46} \mathrm{H}_{74} \mathrm{O}_{14}$ & 873.4927 & -5.0 & {$[\mathrm{M}+\mathrm{Na}]^{+}$} & 729.3908 & Taibaienoside IV & Triterpenoids & NX \\
\hline \multirow[t]{2}{*}{57} & \multirow[t]{2}{*}{21.78} & $\mathrm{C}_{20} \mathrm{H}_{18} \mathrm{O}_{6}$ & 353.1015 & -4.3 & {$[\mathrm{M}-\mathrm{H}]^{-}$} & $\begin{array}{c}252.0419 \\
253.0480\end{array}$ & 8-Prenylkaempferol & Flavonoids & YYH \\
\hline & & $\mathrm{C}_{20} \mathrm{H}_{18} \mathrm{O}_{6}$ & 355.1173 & -0.9 & {$[\mathrm{M}+\mathrm{H}]^{+}$} & $\begin{array}{l}269.0803 \\
299.0550\end{array}$ & 8-Prenylkaempferol & Flavonoids & YYH \\
\hline \multirow[t]{2}{*}{58} & \multirow[t]{2}{*}{21.79} & $\mathrm{C}_{16} \mathrm{H}_{12} \mathrm{O}_{4}$ & 267.0656 & -2.4 & {$[\mathrm{M}-\mathrm{H}]^{-}$} & 252.0419 & Formononetin & Flavonoids & HQ \\
\hline & & $\mathrm{C}_{16} \mathrm{H}_{12} \mathrm{O}_{4}$ & 269.0803 & -2.0 & $\begin{array}{l}{[\mathrm{M}+\mathrm{H}]^{+}} \\
{[\mathrm{M}+\mathrm{Na}]^{+}}\end{array}$ & $\begin{array}{l}118.0413 \\
253.0480\end{array}$ & Formononetin & Flavonoids & HQ \\
\hline 59 & 22.23 & $\mathrm{C}_{17} \mathrm{H}_{16} \mathrm{O}_{5}$ & 301.1080 & 3.3 & {$[\mathrm{M}+\mathrm{H}]^{+}$} & 167.0694 & $\begin{array}{l}\text { (6aR,11aR)-9,10- } \\
\text { dimethoxy-6a,11a- } \\
\text { dihydro-6H- } \\
\text { benzofurano[3,2-c] } \\
\text { chromen-3-ol }\end{array}$ & Flavonoids & HQ \\
\hline \multirow[t]{2}{*}{60} & \multirow[t]{2}{*}{22.33} & $\mathrm{C}_{47} \mathrm{H}_{72} \mathrm{O}_{20}$ & 955.4528 & -1.7 & {$[\mathrm{M}-\mathrm{H}]^{-}$} & $\begin{array}{l}382.1079 \\
645.2166 \\
835.4451 \\
925.4768\end{array}$ & Achyranthoside C & Triterpenoids & NX \\
\hline & & $\mathrm{C}_{47} \mathrm{H}_{72} \mathrm{O}_{20}$ & 995.4167 & -8.2 & {$[\mathrm{M}+\mathrm{K}]^{+}$} & $\begin{array}{l}272.2571 \\
299.0537 \\
383.1132\end{array}$ & Achyranthoside C & Triterpenoids & NX \\
\hline \multirow[t]{2}{*}{61} & \multirow[t]{2}{*}{23.03} & $\mathrm{C}_{32} \mathrm{H}_{38} \mathrm{O}_{14}$ & 645.2190 & 0.2 & $\begin{array}{c}{[\mathrm{M}-\mathrm{H}]^{-}} \\
{[\mathrm{M}+\mathrm{Cl}]^{-}} \\
{[\mathrm{M}+\mathrm{HCOO}]^{-}}\end{array}$ & $\begin{array}{l}223.0282 \\
366.1100\end{array}$ & Sagittatoside B & Flavonoids & YYH \\
\hline & & $\mathrm{C}_{32} \mathrm{H}_{38} \mathrm{O}_{14}$ & 647.2329 & -0.7 & $\begin{array}{l}{[\mathrm{M}+\mathrm{H}]^{+},} \\
{[\mathrm{M}+\mathrm{Na}]^{+}}\end{array}$ & $\begin{array}{l}191.0005 \\
207.0315 \\
313.0700 \\
369.1321\end{array}$ & Sagittatoside B & Flavonoids & YYH \\
\hline 62 & 23.54 & $\mathrm{C}_{17} \mathrm{H}_{20} \mathrm{O}_{6}$ & 343.1163 & 3.2 & {$[\mathrm{M}+\mathrm{Na}]^{+}$} & 131.0491 & $\begin{array}{l}\text { 1,2-Bis(4-hydroxy-3- } \\
\text { methoxyphenyl)-1,3- } \\
\text { propanediol }\end{array}$ & $\begin{array}{l}\text { Phenylpropanoids } \\
\text { (phenylpropionic } \\
\text { acid) }\end{array}$ & YYH \\
\hline \multirow[t]{2}{*}{63} & \multirow[t]{2}{*}{23.89} & $\mathrm{C}_{27} \mathrm{H}_{30} \mathrm{O}_{10}$ & 513.1762 & -0.7 & {$[\mathrm{M}-\mathrm{H}]^{-}$} & $\begin{array}{l}146.9659 \\
217.0478 \\
351.0867 \\
366.1100\end{array}$ & Icariside II & Flavonoids & YYH \\
\hline & & $\mathrm{C}_{27} \mathrm{H}_{30} \mathrm{O}_{10}$ & 515.1900 & -2.2 & $\begin{array}{l}{[\mathrm{M}+\mathrm{H}]^{+}} \\
{[\mathrm{M}+\mathrm{K}]^{+}} \\
{[\mathrm{M}+\mathrm{Na}]^{+}}\end{array}$ & $\begin{array}{l}135.0434 \\
243.0638 \\
313.0698 \\
369.1322\end{array}$ & Icariside II & Flavonoids & YYH \\
\hline 64 & 23.90 & $\mathrm{C}_{21} \mathrm{H}_{20} \mathrm{O}_{6}$ & 369.1321 & -3.2 & {$[\mathrm{M}+\mathrm{H}]^{+}$} & $\begin{array}{l}135.0434 \\
243.0638 \\
313.0698\end{array}$ & Anhydroicaritin & Flavonoids & YYH \\
\hline 65 & 24.53 & $\mathrm{C}_{42} \mathrm{H}_{66} \mathrm{O}_{14}$ & 793.4358 & -2.7 & $\begin{array}{c}{[\mathrm{M}-\mathrm{H}]^{-}} \\
{[\mathrm{M}+\mathrm{HCOO}]^{-}}\end{array}$ & $\begin{array}{c}209.0450 \\
367.1171\end{array}$ & Zingibroside R1 & Triterpenoids & NX \\
\hline
\end{tabular}


TABle 1: Continued.

\begin{tabular}{|c|c|c|c|c|c|c|c|c|c|}
\hline NO. & $\begin{array}{l}\text { Observed } \\
\text { RT (min) }\end{array}$ & Formula & $\begin{array}{l}\text { Observed } \\
\mathrm{m} / \mathrm{z}\end{array}$ & $\begin{array}{l}\text { Mass } \\
\text { error } \\
(\mathrm{ppm})\end{array}$ & Adducts & $\begin{array}{l}\text { Fragment } \\
(+/-)\end{array}$ & Identification & Structure class & Origin \\
\hline 66 & 24.58 & $\mathrm{C}_{19} \mathrm{H}_{30} \mathrm{O}_{9}$ & 437.1603 & 4.3 & {$[\mathrm{M}+\mathrm{Cl}]^{-}$} & 209.0450 & Icariside B3 & $\begin{array}{c}\text { Terpenoids } \\
\text { (Monocyclic } \\
\text { monoterpenoids) }\end{array}$ & YYH \\
\hline 67 & 25.14 & $\mathrm{C}_{41} \mathrm{H}_{64} \mathrm{O}_{12}$ & 793.4391 & 1.5 & {$[\mathrm{M}+\mathrm{HCOO}]^{-}$} & $\begin{array}{l}469.3326 \\
487.3421\end{array}$ & Acetylastragaloside I_qt & Triterpenoids & HQ \\
\hline \multirow[t]{2}{*}{68} & 25.55 & $\mathrm{C}_{20} \mathrm{H}_{18} \mathrm{O}_{5}$ & 337.1068 & -4.0 & {$[\mathrm{M}-\mathrm{H}]^{-}$} & 282.0493 & Yinyanghuo D & Flavonoids & YYH \\
\hline & & $\mathrm{C}_{20} \mathrm{H}_{18} \mathrm{O}_{5}$ & 339.1227 & 0.0 & {$[\mathrm{M}+\mathrm{H}]^{+}$} & 283.0583 & Yinyanghuo D & Flavonoids & YYH \\
\hline \multirow[t]{2}{*}{69} & 25.84 & $\mathrm{C}_{25} \mathrm{H}_{26} \mathrm{O}_{6}$ & 421.1666 & 2.2 & {$[\mathrm{M}-\mathrm{H}]^{-}$} & 351.1189 & Yinyanghuo B & Flavonoids & YYH \\
\hline & & $\mathrm{C}_{25} \mathrm{H}_{26} \mathrm{O}_{6}$ & 423.1829 & 6.5 & {$[\mathrm{M}+\mathrm{H}]^{+}$} & 349.1055 & Yinyanghuo B & Flavonoids & YYH \\
\hline 70 & 26.15 & $\mathrm{C}_{18} \mathrm{H}_{32} \mathrm{O}_{3}$ & 297.2423 & -0.3 & {$[\mathrm{M}+\mathrm{H}]^{+}$} & 119.0855 & $\begin{array}{l}\text { 13-Hydroxy-9,11- } \\
\text { octadecadienoic acid }\end{array}$ & Organic acids & HQ \\
\hline 71 & 26.23 & $\mathrm{C}_{41} \mathrm{H}_{62} \mathrm{O}_{15}$ & 793.4010 & -0.8 & {$[\mathrm{M}-\mathrm{H}]^{-}$} & $\begin{array}{l}631.3795 \\
673.3972\end{array}$ & Achyranthoside C_qt & Triterpenoids & NX \\
\hline 72 & 26.71 & $\mathrm{C}_{45} \mathrm{H}_{56} \mathrm{O}_{23}$ & 1003.2895 & 5.1 & {$[\mathrm{M}+\mathrm{K}]^{+}$} & 313.0709 & Korepimedoside B & Flavonoids & YYH \\
\hline 73 & 26.94 & $\mathrm{C}_{27} \mathrm{H}_{44} \mathrm{O}_{7}$ & 481.3162 & 0.4 & {$[\mathrm{M}+\mathrm{H}]^{+}$} & 251.1605 & Inokosterone & Steroids & NX \\
\hline 74 & 28.62 & $\mathrm{C}_{16} \mathrm{H}_{30} \mathrm{O}_{2}$ & 277.2149 & 4.1 & {$[\mathrm{M}+\mathrm{Na}]^{+}$} & 223.1695 & 2,15-Hexadecanedione & Others & YYH \\
\hline 75 & 29.06 & $\mathrm{C}_{25} \mathrm{H}_{24} \mathrm{O}_{6}$ & 421.1631 & -3.6 & {$[\mathrm{M}+\mathrm{H}]^{+}$} & 367.1135 & Yinyanghuo A & Flavonoids & YYH \\
\hline \multirow[t]{2}{*}{76} & 29.63 & $\mathrm{C}_{21} \mathrm{H}_{20} \mathrm{O}_{5}$ & 351.1228 & -2.9 & {$[\mathrm{M}-\mathrm{H}]^{-}$} & 293.0440 & Artonin U & Flavonoids & YYH \\
\hline & & $\mathrm{C}_{21} \mathrm{H}_{20} \mathrm{O}_{5}$ & 353.1355 & -8.2 & {$[\mathrm{M}+\mathrm{H}]^{+}$} & 297.0770 & Artonin U & Flavonoids & YYH \\
\hline 77 & 29.76 & $\mathrm{C}_{37} \mathrm{H}_{60} \mathrm{O}_{10}$ & 699.3821 & -8.5 & {$[\mathrm{M}+\mathrm{Cl}]^{-}$} & $\begin{array}{l}277.2148 \\
353.2030\end{array}$ & AstragalosideII_qt & Triterpenoids & HQ \\
\hline \multirow[t]{2}{*}{78} & 30.20 & $\mathrm{C}_{25} \mathrm{H}_{26} \mathrm{O}_{5}$ & 405.1700 & -1.8 & {$[\mathrm{M}-\mathrm{H}]^{-}$} & 295.0594 & $8,3^{\prime}$-diprenylapigenin & Flavonoids & YYH \\
\hline & & $\mathrm{C}_{25} \mathrm{H}_{26} \mathrm{O}_{5}$ & 407.1848 & -1.2 & {$[\mathrm{M}+\mathrm{H}]^{+}$} & $\begin{array}{c}149.0233 \\
295.0598 \\
351.1224\end{array}$ & $8,3^{\prime}$-diprenylapigenin & Flavonoids & YYH \\
\hline 79 & 32.96 & $\mathrm{C}_{16} \mathrm{H}_{22} \mathrm{O}_{4}$ & 301.1402 & -2.7 & $\begin{array}{l}{[\mathrm{M}+\mathrm{Na}]^{+}} \\
{[\mathrm{M}+\mathrm{H}]^{+}} \\
{[\mathrm{M}+\mathrm{K}]^{+}}\end{array}$ & 149.0231 & DBP & Others & NX \\
\hline 80 & 37.98 & $\mathrm{C}_{30} \mathrm{H}_{48} \mathrm{O}_{3}$ & 455.3506 & -5.3 & $\begin{array}{c}{[\mathrm{M}-\mathrm{H}]^{-}} \\
{[\mathrm{M}+\mathrm{HCOO}]^{-}}\end{array}$ & 277.2164 & Oleanolic acid & Triterpenoids & $\begin{array}{l}\text { YYH } \\
\text { NX }\end{array}$ \\
\hline 81 & 38.19 & $\mathrm{C}_{18} \mathrm{H}_{30} \mathrm{O}_{2}$ & 279.2309 & -3.4 & {$[\mathrm{M}+\mathrm{H}]^{+}$} & 95.0854 & Linolenic acid & Organic acids & HQ \\
\hline \multirow[t]{2}{*}{82} & 38.31 & $\mathrm{C}_{22} \mathrm{H}_{22} \mathrm{O}_{11}$ & 507.1170 & 5.2 & $\begin{array}{l}{[\mathrm{M}+\mathrm{HCOO}]^{-}} \\
{[\mathrm{M}+\mathrm{Cl}]^{-}}\end{array}$ & 223.0281 & $\begin{array}{l}\text { Rhamnocitrin-3-O- } \\
\text { glucoside }\end{array}$ & Flavonoids & HQ \\
\hline & & $\mathrm{C}_{22} \mathrm{H}_{22} \mathrm{O}_{11}$ & 501.0842 & 9.7 & {$[\mathrm{M}+\mathrm{K}]^{+}$} & $\begin{array}{l}191.0004 \\
207.0315 \\
281.0502\end{array}$ & $\begin{array}{l}\text { Rhamnocitrin-3-O- } \\
\text { glucoside }\end{array}$ & Flavonoids & HQ \\
\hline 83 & 41.16 & $\mathrm{C}_{18} \mathrm{H}_{32} \mathrm{O}_{2}$ & 281.2470 & -1.9 & {$[\mathrm{M}+\mathrm{H}]^{+}$} & 95.0852 & EIC & Organic acids & HQ \\
\hline 84 & 44.22 & $\mathrm{C}_{21} \mathrm{H}_{18} \mathrm{O}_{11}$ & 447.0959 & 8.4 & {$[\mathrm{M}+\mathrm{H}]^{+}$} & $\begin{array}{r}191.0001 \\
115.0034\end{array}$ & Baicalin & Flavonoids & $\mathrm{NX}$ \\
\hline 85 & 46.85 & $\mathrm{C}_{47} \mathrm{H}_{78} \mathrm{O}_{18}$ & 931.5353 & 9.8 & {$[\mathrm{M}+\mathrm{H}]^{+}$} & $\begin{array}{l}147.0651 \\
553.3685\end{array}$ & Asernestioside A & Triterpenoids & HQ \\
\hline \multirow[t]{2}{*}{86} & 46.86 & $\mathrm{C}_{27} \mathrm{H}_{30} \mathrm{O}_{16}$ & 655.1574 & 8.8 & {$[\mathrm{M}+\mathrm{HCOO}]^{-}$} & $\begin{array}{l}91.0223 \\
223.0270\end{array}$ & Rutin & Flavonoids & $\begin{array}{l}\text { YYH } \\
\text { NX } \\
\text { HQ }\end{array}$ \\
\hline & & $\mathrm{C}_{27} \mathrm{H}_{30} \mathrm{O}_{16}$ & 633.1487 & 9.6 & $\begin{array}{l}{[\mathrm{M}+\mathrm{Na}]^{+}} \\
{[\mathrm{M}+\mathrm{H}]^{+}} \\
{[\mathrm{M}+\mathrm{K}]^{+}}\end{array}$ & $\begin{array}{c}115.0034 \\
147.0651 \\
190.9999 \\
207.0309 \\
355.068\end{array}$ & Rutin & Flavonoids & $\begin{array}{l}\text { YYH } \\
\text { NX } \\
\text { HQ }\end{array}$ \\
\hline 87 & 48.41 & $\mathrm{C}_{49} \mathrm{H}_{80} \mathrm{O}_{19}$ & 1011.4944 & 1.8 & {$[\mathrm{M}+\mathrm{K}]^{+}$} & $\begin{array}{c}147.0656 \\
184.0726 \\
621.3171\end{array}$ & Asernestioside B & Triterpenoids & HQ \\
\hline 88 & 49.83 & $\mathrm{C}_{17} \mathrm{H}_{14} \mathrm{O}_{6}$ & 315.0886 & 7.2 & {$[\mathrm{M}+\mathrm{H}]^{+}$} & $\begin{array}{l}207.0301 \\
281.0486\end{array}$ & Jaranol & Flavonoids & HQ \\
\hline 89 & 50.62 & $\mathrm{C}_{35} \mathrm{H}_{60} \mathrm{O}_{6}$ & 621.4377 & 0.8 & $\begin{array}{c}{[\mathrm{M}+\mathrm{HCOO}]^{-}} \\
{[\mathrm{M}+\mathrm{Cl}]^{-}}\end{array}$ & 277.2198 & Daucosterol & Steroids & $\begin{array}{l}\text { YYH } \\
\text { NX }\end{array}$ \\
\hline 90 & 52.31 & $\mathrm{C}_{33} \mathrm{H}_{42} \mathrm{O}_{15}$ & 717.2087 & -9.5 & {$[\mathrm{M}+\mathrm{K}]^{+}$} & $\begin{array}{l}221.0835 \\
429.0876\end{array}$ & Wanepimedoside A & Flavonoids & YYH \\
\hline
\end{tabular}


TABle 1: Continued.

\begin{tabular}{|c|c|c|c|c|c|c|c|c|c|}
\hline NO. & $\begin{array}{l}\text { Observed } \\
\text { RT (min) }\end{array}$ & Formula & $\begin{array}{c}\text { Observed } \\
\mathrm{m} / \mathrm{z}\end{array}$ & $\begin{array}{l}\text { Mass } \\
\text { error } \\
(\mathrm{ppm})\end{array}$ & Adducts & $\begin{array}{l}\text { Fragment } \\
\quad(+/-)\end{array}$ & Identification & Structure class & Origin \\
\hline 91 & 57.66 & $\mathrm{C}_{20} \mathrm{H}_{36} \mathrm{O}_{2}$ & 309.2763 & -8.2 & {$[\mathrm{M}+\mathrm{H}]^{+}$} & 89.0612 & $\begin{array}{l}\text { Linoelaidyl acetate } \\
5^{\prime} \text {-Hydroxyiso- }\end{array}$ & Others & YYH \\
\hline 92 & 57.75 & $\mathrm{C}_{29} \mathrm{H}_{38} \mathrm{O}_{16}$ & 665.2115 & 9.5 & {$[\mathrm{M}+\mathrm{Na}]^{+}$} & 133.0854 & $\begin{array}{l}\text { muronulatol-2 } 2^{\prime}, 5^{\prime} \text {-di-O- } \\
\text { glucoside }\end{array}$ & Saccharide & HQ \\
\hline 93 & 57.90 & $\mathrm{C}_{29} \mathrm{H}_{48} \mathrm{O}$ & 413.3796 & 4.3 & {$[\mathrm{M}+\mathrm{H}]^{+}$} & 95.0869 & Stigmasterol & Steroids & $\mathrm{NX}$ \\
\hline
\end{tabular}

YYH: Herba Epimedii; NX: Radix Achyranthis Bidentatae; HQ: Radix Astragali; ${ }^{*}$ Compared with a reference standard.

\subsection{Identification and Analysis of Chemical Constituents in} Rat Serum. When analyzing the rat serum samples of GSL, the selected database was a self-built database constructed from analyzed and confirmed GSL in vitro compound information. The analysis steps of GSL in vivo components were the same as in vitro processing. The results showed that there were 20 chemical constituents in rat serum, including10 kinds of flavonoids, 4 kinds of triterpenoids, 2 kinds of phenylpropanoids, 1 kind of steroid, 1 kind of organic acid, and 2 others. Among them, 12 compounds were from Herba Epimedii, 8 compounds from Radix Achyranthis Bidentatae, and 2 compounds from Radix Astragali. At the same time, rutin is a common compound of three Chinese herbal medicines. Five compounds were identified by the standard substance. The specific information is shown in Table 2 and Figure S2.

3.4. Identification and Analysis of Metabolites in Rat Serum. After the chemical constituents from the GSL get into the body, some components exist in the form of a prototype, but most of them are structurally modified based on the original components, such as oxidation, reduction, and hydration. With the unique data postprocessing function of UNIFI, we analyzed the possible metabolites based on the screened prototype compounds in vivo and finally obtained 42 metabolites in rat serum through artificial screening. After analysis, these 42 metabolites mainly undergo oxidation, hydration, reduction, dehydration, glutathione S-conjugation, and acetylcysteine conjugation reactions based on the prototype compounds. The detailed information of the prototype, metabolite name, molecular weight, and molecular formula is given in Table 3.

\subsection{Analysis of GSL by UPLC-Q-TOF-MS}

3.5.1. Flavonoids. Flavonoids and their glycosides are the main ingredients and the major bioactive components of GSL. In this study, 3 flavones, 41 flavonols, 5 isoflavones, 1 isoflavanone, and 1 flavanol were determined by the matching of the mass spectrometry data with the UNIFI analysis platform.

For flavonoid glycosides, the glycosidic bonds connected by oxygen atoms could be cleaved in both positive and negative ion modes, and most of them were characterized by neutral losses such as $162 \mathrm{Da}(\mathrm{Glc})$ and $146 \mathrm{Da}$ (Rha) [24]. It was difficult to directly remove the glycosyl groups connected by carbon-glycosidic bonds and often produce $[\mathrm{M}+\mathrm{H}-120]^{+}$fragment [25]. As we all know, the main cleavage behavior of flavonoid aglycones was the retro Diels-Alder reaction (RDA) cleavage pathway and the loss of free radicals and/or small molecules (such as $\mathrm{CH}_{3}, \mathrm{CO}$, and $\mathrm{CO}_{2}$ ) [26]. By comparing the retention time and fragmentation patterns with standard substance, peaks 14, $31,33,34,35,37$, and 41 in Figure 1 were exactly identified as calycosin-7-glucoside, ononin, calycosin, epimedin A, epimedin $\mathrm{B}$, epimedin $\mathrm{C}$, and icariin, respectively. Here, we took epimedin $\mathrm{C}$ and icariin as examples to depict the fragmentation mode of these ingredients.

Epimedin C showed quasi-molecular ion $[\mathrm{M}+\mathrm{H}]^{+}$at $\mathrm{m} / z$ 823.3014 in positive ion mode and yielded fragment ions at $\mathrm{m} / z 531.1852$ and 515.1931 by losses of 2 molecules of rhamnose and a molecule of ORha-Rha group, respectively. Then, the ion at $\mathrm{m} / z 515.1931$ loses a molecule of Glc to generate an ion at $\mathrm{m} / z 369.1322$, and the ion at $\mathrm{m} / z 369.1322$ further loses a functional group of $\mathrm{C}_{4} \mathrm{H}_{8}$ to produce ion at $\mathrm{m} /$ $z$ 313.0699. The mass spectrogram and possible fragmentation pathways of epimedin $\mathrm{C}$ in positive ion mode are shown in Figure 3.

In the positive ion mode, the mass-to-charge ratio of the quasi-molecular ion peak of compound 41 was 677.2433 $[\mathrm{M}+\mathrm{H}]^{+}$as shown in Table 1 . The mass-to-charge ratio of the fragment ions produced by the precursor ions were 531.1851 $[\mathrm{M}+\mathrm{H}-\mathrm{Rha}]^{+}, \quad 369.1321 \quad[\mathrm{M}+\mathrm{H}-\mathrm{Glc}]^{+}, \quad$ and 313.0698 $\left[\mathrm{M}+\mathrm{H}-\mathrm{C}_{4} \mathrm{H}_{8}\right]^{+}$, which are consistent with those of icariin. The mass spectrogram and potential fragmentation pathways of icariin in positive ion mode are shown in Figure S3.

3.5.2. Terpenoids. The terpenoids in GSL primarily included monocyclic monoterpenoids, cycloartane-type tetracyclic triterpenoids, and oleanane-type pentacyclic triterpenoids. Among them, the number of the above categories in GSL was 3,4 , and 11 , respectively.

Triterpene saponins in GSL mainly exist in the form of aglycones binding with sugars, such as glucose, rhamnose, and xylose. In mass spectrometric analysis, triterpenoid saponins are mostly in the form of de-sugar or continuous de-sugar fragment ions [27]. Such compounds also had the loss of $\mathrm{CO}_{2}, \mathrm{COOH}, \mathrm{CH}_{2} \mathrm{OH}$, and other complex groups. Compound 49 gave a deprotonated molecule $[\mathrm{M}-\mathrm{H}]^{-}$at $\mathrm{m} / z$ 1117.5026 and produced predominant fragment ions at $\mathrm{m} / z$ $997.4956\left[\mathrm{M}-\mathrm{H}-\mathrm{COOH}-\mathrm{CH}_{2} \mathrm{OH}-\mathrm{CO}_{2}\right]^{-}$and $\mathrm{m} / z 631.2021$ $\left[\mathrm{M}-\mathrm{H}-\mathrm{C}_{2} \mathrm{H}_{2} \mathrm{O}-2 \mathrm{Glc}\right]^{-}$in negative ion mode (Figure 4). It 


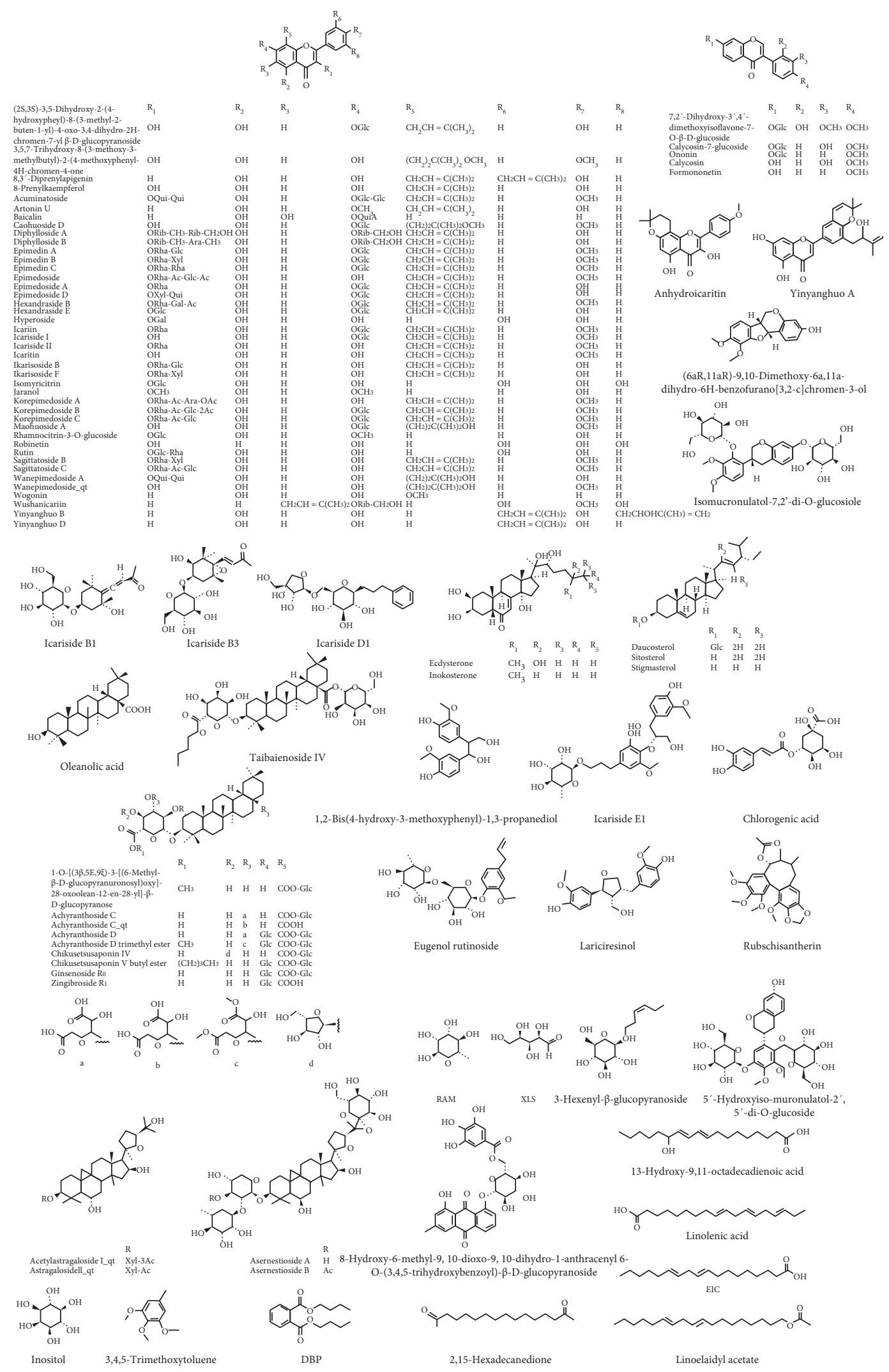

Figure 2: Chemical structures of compounds identified in GSL. 
TABLE 2: The chemical constituents of the GSL blood prototype identified by UPLC-Q-TOF-MS.

\begin{tabular}{|c|c|c|c|c|c|c|c|c|c|}
\hline NO. & $\begin{array}{l}\text { Observed } \\
\text { RT (min) }\end{array}$ & Formula & $\begin{array}{l}\text { Observed } \\
\mathrm{m} / \mathrm{z}\end{array}$ & $\begin{array}{l}\text { Mass } \\
\text { error } \\
(\mathrm{ppm}) \\
\end{array}$ & Adducts & $\begin{array}{l}\text { Fragment } \\
(+/-)\end{array}$ & Identification & Structure class & Origin \\
\hline 1 & 5.26 & $\mathrm{C}_{16} \mathrm{H}_{18} \mathrm{O}_{9}$ & 353.0846 & -9.2 & {$[\mathrm{M}-\mathrm{H}]^{-}$} & 93.0350 & Chlorogenic acid & $\begin{array}{c}\text { Phenylpropanoids } \\
\text { (phenylpropionic } \\
\text { acid) }\end{array}$ & YYH \\
\hline 2 & 8.40 & $\mathrm{C}_{27} \mathrm{H}_{44} \mathrm{O}_{7}$ & 481.3201 & 8.5 & {$[\mathrm{M}+\mathrm{H}]^{+}$} & $\begin{array}{l}175.1189 \\
288.2025\end{array}$ & Ecdysterone* & Steroids & NX \\
\hline 3 & 12.30 & $\mathrm{C}_{43} \mathrm{H}_{68} \mathrm{O}_{14}$ & 853.4568 & -2.8 & {$[\mathrm{M}+\mathrm{HCOO}]^{-}$} & $\begin{array}{l}137.0231 \\
312.1296\end{array}$ & $\begin{array}{c}\text { 1-O- }\{(3 \beta, 5 \xi, 9 \xi)-3-[(6- \\
\text { Methyl- } \beta \text {-D- } \\
\text { glucopyranuronosyl) } \\
\text { oxy]-28-oxoolean-12- } \\
\text { en-28-yl }\}-\beta-D- \\
\text { glucopyranose }\end{array}$ & Triterpenoids & NX \\
\hline 4 & 12.72 & $\mathrm{C}_{38} \mathrm{H}_{48} \mathrm{O}_{20}$ & 823.2644 & -2.7 & {$[\mathrm{M}-\mathrm{H}]^{-}$} & $\begin{array}{l}214.9992 \\
312.1294\end{array}$ & Diphylloside A & Flavonoids & YYH \\
\hline 5 & 13.01 & $\mathrm{C}_{37} \mathrm{H}_{46} \mathrm{O}_{19}$ & 793.2537 & -3.0 & {$[\mathrm{M}-\mathrm{H}]^{-}$} & 631.2019 & Epimedoside D & Flavonoids & YYH \\
\hline 6 & 13.34 & $\mathrm{C}_{32} \mathrm{H}_{38} \mathrm{O}_{15}$ & 661.2083 & -8.3 & $\begin{array}{c}{[\mathrm{M}-\mathrm{H}]^{-}} \\
{[\mathrm{M}+\mathrm{HCOO}]^{-}}\end{array}$ & 353.0997 & Epimedoside A & Flavonoids & YYH \\
\hline 7 & 17.58 & $\mathrm{C}_{39} \mathrm{H}_{50} \mathrm{O}_{19}$ & 883.2807 & -8.0 & {$[\mathrm{M}+\mathrm{HCOO}]^{-}$} & 675.2247 & Epimedin $\mathrm{A}^{*}$ & Flavonoids & YYH \\
\hline \multirow[t]{2}{*}{8} & \multirow[t]{2}{*}{18.44} & $\mathrm{C}_{38} \mathrm{H}_{48} \mathrm{O}_{18}$ & 853.2745 & -3.2 & $\begin{array}{c}{[\mathrm{M}+\mathrm{HCOO}]^{-}} \\
{[\mathrm{M}+\mathrm{Cl}]^{-}}\end{array}$ & $\begin{array}{l}252.0406 \\
366.1081 \\
645.2177\end{array}$ & Epimedin $\mathrm{B}^{*}$ & Flavonoids & YYH \\
\hline & & $\mathrm{C}_{38} \mathrm{H}_{48} \mathrm{O}_{18}$ & 809.2861 & -0.2 & {$[\mathrm{M}+\mathrm{H}]^{+}$} & $\begin{array}{c}369.1333 \\
531.1842\end{array}$ & Epimedin $\mathrm{B}^{*}$ & Flavonoids & YYH \\
\hline \multirow[t]{2}{*}{9} & \multirow[t]{2}{*}{18.92} & $\mathrm{C}_{39} \mathrm{H}_{50} \mathrm{O}_{19}$ & 867.2915 & -1.6 & {$[\mathrm{M}+\mathrm{HCOO}]^{-}$} & $\begin{array}{l}366.1059 \\
659.2331\end{array}$ & Epimedin $\mathrm{C}^{*}$ & Flavonoids & YYH \\
\hline & & $\mathrm{C}_{39} \mathrm{H}_{50} \mathrm{O}_{19}$ & 823.3031 & 1.5 & {$[\mathrm{M}+\mathrm{H}]^{+}$} & $\begin{array}{l}136.0765 \\
531.1840\end{array}$ & Epimedin $C^{*}$ & Flavonoids & YYH \\
\hline \multirow[t]{2}{*}{10} & \multirow[t]{2}{*}{19.20} & $\mathrm{C}_{33} \mathrm{H}_{40} \mathrm{O}_{15}$ & 721.2341 & -1.2 & $\begin{array}{c}{[\mathrm{M}+\mathrm{HCOO}]^{-}} \\
{[\mathrm{M}+\mathrm{Cl}]^{-}}\end{array}$ & 367.1163 & Icariin* & Flavonoids & YYH \\
\hline & & $\mathrm{C}_{33} \mathrm{H}_{40} \mathrm{O}_{15}$ & 677.2413 & -4.0 & {$[\mathrm{M}+\mathrm{H}]^{+}$} & $\begin{array}{l}313.0683 \\
369.1314 \\
531.1844\end{array}$ & Icariin* & Flavonoids & YYH \\
\hline 11 & 19.45 & $\mathrm{C}_{25} \mathrm{H}_{30} \mathrm{O}_{8}$ & 481.1812 & -4.3 & {$[\mathrm{M}+\mathrm{Na}]^{+}$} & 97.0644 & Rubschisantherin & $\begin{array}{c}\text { Phenylpropanoids } \\
\text { (lignans) }\end{array}$ & NX \\
\hline 12 & 21.12 & $\mathrm{C}_{53} \mathrm{H}_{82} \mathrm{O}_{25}$ & 1117.5037 & -3.2 & {$[\mathrm{M}-\mathrm{H}]^{-}$} & $\begin{array}{c}217.0817 \\
365.2313 \\
413.1985 \\
585.2846 \\
785.4175\end{array}$ & Achyranthoside D & Triterpenoids & NX \\
\hline 13 & 21.21 & $\mathrm{C}_{48} \mathrm{H}_{76} \mathrm{O}_{19}$ & 955.4937 & 3.0 & {$[\mathrm{M}-\mathrm{H}]^{-}$} & $\begin{array}{l}413.1985 \\
585.2846 \\
785.4175\end{array}$ & Ginsenoside Ro* & Triterpenoids & NX \\
\hline 14 & 21.92 & $\mathrm{C}_{47} \mathrm{H}_{72} \mathrm{O}_{20}$ & 955.4622 & 8.2 & {$[\mathrm{M}-\mathrm{H}]^{-}$} & $\begin{array}{l}353.2213 \\
405.2627\end{array}$ & Achyranthoside C & Triterpenoids & NX \\
\hline 15 & 22.99 & $\mathrm{C}_{32} \mathrm{H}_{38} \mathrm{O}_{14}$ & 645.2157 & -4.9 & {$[\mathrm{M}-\mathrm{H}]^{-}$} & 223.0270 & Sagittatoside B & Flavonoids & YYH \\
\hline 16 & 23.89 & $\begin{array}{l}\mathrm{C}_{27} \mathrm{H}_{30} \mathrm{O}_{10} \\
\mathrm{C}_{27} \mathrm{H}_{30} \mathrm{O}_{10}\end{array}$ & $\begin{array}{l}513.1739 \\
515.1908\end{array}$ & $\begin{array}{l}-5.3 \\
-0.8\end{array}$ & $\begin{array}{l}{[\mathrm{M}-\mathrm{H}]^{-}} \\
{[\mathrm{M}+\mathrm{H}]^{+}}\end{array}$ & $\begin{array}{l}366.1082 \\
313.0671\end{array}$ & $\begin{array}{l}\text { Icariside II } \\
\text { Icariside II }\end{array}$ & $\begin{array}{l}\text { Flavonoids } \\
\text { Flavonoids }\end{array}$ & $\begin{array}{l}\text { YYH } \\
\text { YYH }\end{array}$ \\
\hline 17 & 28.61 & $\mathrm{C}_{16} \mathrm{H}_{30} \mathrm{O}_{2}$ & 277.2140 & 0.6 & $\begin{array}{l}{[\mathrm{M}+\mathrm{Na}]^{+}} \\
{[\mathrm{M}+\mathrm{Na}]^{+},}\end{array}$ & 223.1695 & 2,15-Hexadecanedione & Others & YYH \\
\hline 18 & 32.96 & $\mathrm{C}_{16} \mathrm{H}_{22} \mathrm{O}_{4}$ & 301.1382 & -9.4 & $\begin{array}{l}{[\mathrm{M}+\mathrm{H}]^{+},} \\
{[\mathrm{M}+\mathrm{K}]^{+}}\end{array}$ & 149.0216 & DBP & Others & NX \\
\hline 19 & 38.18 & $\mathrm{C}_{18} \mathrm{H}_{30} \mathrm{O}_{2}$ & 279.2323 & 1.7 & {$[\mathrm{M}+\mathrm{H}]^{+}$} & 95.0865 & Linolenic acid & Organic acids & $\begin{array}{l}\text { HQ } \\
\text { YYH }\end{array}$ \\
\hline \multirow[t]{2}{*}{20} & \multirow[t]{2}{*}{46.60} & $\mathrm{C}_{27} \mathrm{H}_{30} \mathrm{O}_{16}$ & 645.1256 & 4.4 & {$[\mathrm{M}+\mathrm{Cl}]^{-}$} & 168.0420 & Rutin & Flavonoids & $\begin{array}{l}\text { NX } \\
\text { HQ }\end{array}$ \\
\hline & & $\mathrm{C}_{27} \mathrm{H}_{30} \mathrm{O}_{16}$ & 633.1444 & 2.8 & $\begin{array}{c}{[\mathrm{M}+\mathrm{Na}]^{+}} \\
{[\mathrm{M}+\mathrm{K}]^{+}}\end{array}$ & 207.0296 & Rutin & Flavonoids & $\begin{array}{l}\text { YYH } \\
\text { NX } \\
\text { HQ }\end{array}$ \\
\hline
\end{tabular}


TABLE 3: Identification of the metabolites from GSL in vivo.

\begin{tabular}{|c|c|c|c|c|c|c|}
\hline NO. & Observed RT (min) & Formula & Observed $\mathrm{m} / \mathrm{z}$ & Mass error (ppm) & Adducts & Identification \\
\hline M1 & 4.8 & $\mathrm{C}_{39} \mathrm{H}_{52} \mathrm{O}_{20}$ & 875.2792 & 5.2 & {$[\mathrm{M}+\mathrm{Cl}]^{-}$} & Epimedin $\mathrm{C}+\mathrm{H}_{2}+\mathrm{O}$ \\
\hline M2 & 7.4 & $\mathrm{C}_{28} \mathrm{H}_{47} \mathrm{~N}_{3} \mathrm{O}_{9} \mathrm{~S}$ & 646.2978 & -5.8 & {$[\mathrm{M}+\mathrm{HCOO}]^{-}$} & Linolenic acid $+\mathrm{H}_{2} \mathrm{O}+\mathrm{C}_{10} \mathrm{H}_{15} \mathrm{~N}_{3} \mathrm{O}_{6} \mathrm{~S}$ \\
\hline M3 & 7.57 & $\mathrm{C}_{32} \mathrm{H}_{55} \mathrm{NO}_{11} \mathrm{~S}$ & 696.3213 & 3.2 & {$[\mathrm{M}+\mathrm{Cl}]^{-}$} & Ecdysterone $+\mathrm{H}_{2}+\mathrm{H}_{2} \mathrm{O}+\mathrm{C}_{5} \mathrm{H}_{7} \mathrm{NO}_{3} \mathrm{~S}$ \\
\hline M4 & 7.72 & $\mathrm{C}_{27} \mathrm{H}_{45} \mathrm{O}_{12} \mathrm{P}$ & 591.2631 & 9.3 & {$[\mathrm{M}-\mathrm{H}]^{-}$} & Ecdysterone $+2 \mathrm{x}(+\mathrm{O})+\mathrm{HPO}_{3}$ \\
\hline M5 & 7.73 & $\mathrm{C}_{27} \mathrm{H}_{47} \mathrm{O}_{12} \mathrm{P}$ & 629.2539 & 6.4 & {$[\mathrm{M}+\mathrm{Cl}]^{-}$} & Ecdysterone $+\mathrm{O}+\mathrm{H}_{2} \mathrm{O}+\mathrm{HPO}_{3}$ \\
\hline M6 & 7.89 & $\mathrm{C}_{24} \mathrm{H}_{31} \mathrm{O}_{9} \mathrm{P}$ & 493.1677 & 8.9 & {$[\mathrm{M}-\mathrm{H}]^{-}$} & Rubschisantherin $-\mathrm{COO}+\mathrm{HPO}_{3}$ \\
\hline M7 & 8.24 & $\mathrm{C}_{25} \mathrm{H}_{30} \mathrm{O}_{10}$ & 535.1793 & -5.2 & {$[\mathrm{M}+\mathrm{HCOO}]^{-}$} & Rubschisantherin $+2 \mathrm{x}(+\mathrm{O})$ \\
\hline M8 & 8.41 & $\mathrm{C}_{37} \mathrm{H}_{59} \mathrm{~N}_{3} \mathrm{O}_{15} \mathrm{~S}$ & 852.3433 & 8.4 & {$[\mathrm{M}+\mathrm{Cl}]^{-}$} & Ecdysterone $+2 \mathrm{x}(+\mathrm{O})+\mathrm{C}_{10} \mathrm{H}_{15} \mathrm{~N}_{3} \mathrm{O}_{6} \mathrm{~S}$ \\
\hline M9 & 14.8 & $\mathrm{C}_{24} \mathrm{H}_{30} \mathrm{O}_{7}$ & 429.193 & 2.7 & {$[\mathrm{M}-\mathrm{H}]^{-}$} & Rubschisantherin $+\mathrm{O}-\mathrm{COO}$ \\
\hline M10 & 16.62 & $\mathrm{C}_{33} \mathrm{H}_{36} \mathrm{O}_{21}$ & 803.1385 & -7.2 & {$[\mathrm{M}+\mathrm{Cl}]^{-}$} & Rutin $-\mathrm{H}_{2} \mathrm{O}+\mathrm{C}_{6} \mathrm{H}_{8} \mathrm{O}_{6}$ \\
\hline M11 & 19.74 & $\mathrm{C}_{39} \mathrm{H}_{48} \mathrm{O}_{19}$ & 865.2694 & -8.9 & {$[\mathrm{M}+\mathrm{HCOO}]^{-}$} & Epimedin $\mathrm{A}-\mathrm{H}_{2} \mathrm{O}$ \\
\hline M12 & 19.79 & $\mathrm{C}_{38} \mathrm{H}_{46} \mathrm{O}_{20}$ & 821.2456 & -6.6 & {$[\mathrm{M}-\mathrm{H}]^{-}$} & Sagittatoside $B+\mathrm{C}_{6} \mathrm{H}_{8} \mathrm{O}_{6}$ \\
\hline M13 & 20.03 & $\mathrm{C}_{24} \mathrm{H}_{30} \mathrm{O}_{6}$ & 413.199 & 4.9 & {$[\mathrm{M}-\mathrm{H}]^{-}$} & Rubschisantherin - COO \\
\hline M14 & 20.04 & $\mathrm{C}_{38} \mathrm{H}_{46} \mathrm{O}_{18}$ & 835.2618 & -5.8 & {$[\mathrm{M}+\mathrm{HCOO}]^{-}$} & Epimedin $\mathrm{B}-\mathrm{H}_{2} \mathrm{O}$ \\
\hline M15 & 22.34 & $\mathrm{C}_{27} \mathrm{H}_{46} \mathrm{O}_{8}$ & 497.3131 & 2.2 & {$[\mathrm{M}-\mathrm{H}]^{-}$} & Ecdysterone $+\mathrm{H}_{2} \mathrm{O}$ \\
\hline M16 & 23.09 & $\mathrm{C}_{33} \mathrm{H}_{40} \mathrm{O}_{14}$ & 659.2317 & -4.3 & {$[\mathrm{M}-\mathrm{H}]^{-}$} & Icariin $+\mathrm{H}_{2}-\mathrm{H}_{2} \mathrm{O}$ \\
\hline M17 & 24.63 & $\mathrm{C}_{32} \mathrm{H}_{51} \mathrm{NO}_{12} \mathrm{~S}$ & 672.312 & 9.1 & {$[\mathrm{M}-\mathrm{H}]^{-}$} & Ecdysterone $+2 x(+\mathrm{O})+\mathrm{C}_{5} \mathrm{H}_{7} \mathrm{NO}_{3} \mathrm{~S}$ \\
\hline M18 & 26.05 & $\mathrm{C}_{18} \mathrm{H}_{34} \mathrm{O}_{4}$ & 313.2376 & -2.9 & {$[\mathrm{M}-\mathrm{H}]^{-}$} & Linolenic acid $+2 \mathrm{x}\left(+\mathrm{H}_{2} \mathrm{O}\right)$ \\
\hline M19 & 27.3 & $\mathrm{C}_{18} \mathrm{H}_{31} \mathrm{O}_{6} \mathrm{P}$ & 409.1519 & -8.1 & {$[\mathrm{M}+\mathrm{Cl}]^{-}$} & Linolenic acid $+\mathrm{O}+\mathrm{HPO}_{3}$ \\
\hline M20 & 27.48 & $\mathrm{C}_{27} \mathrm{H}_{49} \mathrm{O}_{11} \mathrm{P}$ & 579.2972 & 5.5 & {$[\mathrm{M}-\mathrm{H}]^{-}$} & Ecdysterone $+\mathrm{H}_{2}+\mathrm{H}_{2} \mathrm{O}+\mathrm{HPO}_{3}$ \\
\hline M21 & 27.59 & $\mathrm{C}_{28} \mathrm{H}_{49} \mathrm{~N}_{3} \mathrm{O}_{8} \mathrm{~S}$ & 586.3145 & -4.1 & {$[\mathrm{M}-\mathrm{H}]^{-}$} & Linolenic acid $+2 \mathrm{x}\left(+\mathrm{H}_{2}\right)+\mathrm{C}_{10} \mathrm{H}_{15} \mathrm{~N}_{3} \mathrm{O}_{6} \mathrm{~S}$ \\
\hline M22 & 27.85 & $\mathrm{C}_{28} \mathrm{H}_{47} \mathrm{~N}_{3} \mathrm{O}_{8} \mathrm{~S}$ & 630.3034 & -5.3 & {$[\mathrm{M}+\mathrm{HCOO}]^{-}$} & Linolenic acid $+\mathrm{H}_{2}+\mathrm{C}_{10} \mathrm{H}_{15} \mathrm{~N}_{3} \mathrm{O}_{6} \mathrm{~S}$ \\
\hline M23 & 27.88 & $\mathrm{C}_{18} \mathrm{H}_{30} \mathrm{O}_{4}$ & 309.2043 & -9.1 & {$[\mathrm{M}-\mathrm{H}]^{-}$} & Linolenic acid $+2 \mathrm{x}(+\mathrm{O})$ \\
\hline M24 & 28.66 & $\mathrm{C}_{27} \mathrm{H}_{46} \mathrm{O}_{7}$ & 527.3212 & -2.7 & {$[\mathrm{M}+\mathrm{HCOO}]^{-}$} & Ecdysterone $+\mathrm{H}_{2}$ \\
\hline M25 & 28.82 & $\mathrm{C}_{27} \mathrm{H}_{44} \mathrm{O}_{9}$ & 557.2997 & 5.4 & {$[\mathrm{M}+\mathrm{HCOO}]^{-}$} & Ecdysterone $+2 \mathrm{x}(+\mathrm{O})$ \\
\hline M26 & 28.83 & $\mathrm{C}_{28} \mathrm{H}_{47} \mathrm{~N}_{3} \mathrm{O}_{10} \mathrm{~S}$ & 616.2894 & -2.7 & {$[\mathrm{M}-\mathrm{H}]^{-}$} & Linolenic acid $+\mathrm{O}+\mathrm{H}_{2} \mathrm{O}+\mathrm{C}_{10} \mathrm{H}_{15} \mathrm{~N}_{3} \mathrm{O}_{6} \mathrm{~S}$ \\
\hline M27 & 28.86 & $\mathrm{C}_{32} \mathrm{H}_{55} \mathrm{NO}_{10} \mathrm{~S}$ & 680.3184 & -8.6 & {$[\mathrm{M}+\mathrm{Cl}]^{-}$} & Ecdysterone $+2 \mathrm{x}\left(+\mathrm{H}_{2}\right)+\mathrm{C}_{5} \mathrm{H}_{7} \mathrm{NO}_{3} \mathrm{~S}$ \\
\hline M28 & 28.97 & $\mathrm{C}_{32} \mathrm{H}_{51} \mathrm{NO}_{11} \mathrm{~S}$ & 656.3155 & 6.9 & {$[\mathrm{M}-\mathrm{H}]^{-}$} & Ecdysterone $+\mathrm{O}+\mathrm{C}_{5} \mathrm{H}_{7} \mathrm{NO}_{3} \mathrm{~S}$ \\
\hline M29 & 29.08 & $\mathrm{C}_{27} \mathrm{H}_{44} \mathrm{O}_{8}$ & 531.2733 & 0.5 & {$[\mathrm{M}+\mathrm{Cl}]^{-}$} & Ecdysterone $+\mathrm{O}$ \\
\hline M30 & 30.24 & $\mathrm{C}_{18} \mathrm{H}_{32} \mathrm{O}_{3}$ & 295.2255 & -8 & {$[\mathrm{M}-\mathrm{H}]^{-}$} & Linolenic acid $+\mathrm{H}_{2} \mathrm{O}$ \\
\hline M31 & 31.03 & $\mathrm{C}_{23} \mathrm{H}_{41} \mathrm{NO}_{7} \mathrm{~S}$ & 520.2627 & 7.8 & {$[\mathrm{M}+\mathrm{HCOO}]^{-}$} & Linolenic acid $+2 \mathrm{x}\left(+\mathrm{H}_{2} \mathrm{O}\right)+\mathrm{C}_{5} \mathrm{H}_{7} \mathrm{NO}_{3} \mathrm{~S}$ \\
\hline M32 & 32.47 & $\mathrm{C}_{32} \mathrm{H}_{47} \mathrm{NO}_{8} \mathrm{~S}$ & 640.2711 & -0.7 & {$[\mathrm{M}+\mathrm{Cl}]^{-}$} & Ecdysterone $+2 \mathrm{x}\left(-\mathrm{H}_{2} \mathrm{O}\right)+\mathrm{C}_{5} \mathrm{H}_{7} \mathrm{NO}_{3} \mathrm{~S}$ \\
\hline M33 & 34.78 & $\mathrm{C}_{18} \mathrm{H}_{32} \mathrm{O}_{4}$ & 347.1975 & -5.8 & {$[\mathrm{M}+\mathrm{Cl}]^{-}$} & Linolenic acid $+\mathrm{O}+\mathrm{H}_{2} \mathrm{O}$ \\
\hline M34 & 34.9 & $\mathrm{C}_{32} \mathrm{H}_{49} \mathrm{NO}_{10} \mathrm{~S}$ & 638.2981 & -3.6 & {$[\mathrm{M}-\mathrm{H}]^{-}$} & Ecdysterone $+\mathrm{O}-\mathrm{H}_{2} \mathrm{O}+\mathrm{C}_{5} \mathrm{H}_{7} \mathrm{NO}_{3} \mathrm{~S}$ \\
\hline M35 & 37.62 & $\mathrm{C}_{23} \mathrm{H}_{30} \mathrm{O}_{4}$ & 369.2044 & -7.4 & {$[\mathrm{M}-\mathrm{H}]^{-}$} & Rubschisantherin $+2 x(-\mathrm{COO})$ \\
\hline M36 & 38.5 & $\mathrm{C}_{34} \mathrm{H}_{45} \mathrm{~N}_{3} \mathrm{O}_{12} \mathrm{~S}$ & 754.2396 & -3 & {$[\mathrm{M}+\mathrm{Cl}]^{-}$} & Rubschisantherin- $\mathrm{COO}+\mathrm{C}_{10} \mathrm{H}_{15} \mathrm{~N}_{3} \mathrm{O}_{6} \mathrm{~S}$ \\
\hline M37 & 40.72 & $\mathrm{C}_{33} \mathrm{H}_{40} \mathrm{O}_{17}$ & 753.2191 & -7.5 & {$[\mathrm{M}+\mathrm{HCOO}]^{-}$} & Icariin $+2 x(+O)$ \\
\hline M38 & 41.15 & $\mathrm{C}_{33} \mathrm{H}_{52} \mathrm{O}_{15}$ & 733.3252 & -5 & {$[\mathrm{M}+\mathrm{HCOO}]^{-}$} & Ecdysterone $+2 \mathrm{x}(+\mathrm{O})+\mathrm{C}_{6} \mathrm{H}_{8} \mathrm{O}_{6}$ \\
\hline M39 & 44.28 & $\mathrm{C}_{33} \mathrm{H}_{56} \mathrm{O}_{15}$ & 737.3607 & 0.7 & {$[\mathrm{M}+\mathrm{HCOO}]^{-}$} & Ecdysterone $+2 \mathrm{x}\left(+\mathrm{H}_{2} \mathrm{O}\right)+\mathrm{C}_{6} \mathrm{H}_{8} \mathrm{O}_{6}$ \\
\hline M40 & 51.66 & $\mathrm{C}_{33} \mathrm{H}_{40} \mathrm{O}_{23}$ & 803.1956 & 8.5 & {$[\mathrm{M}-\mathrm{H}]^{-}$} & Rutin $+\mathrm{H}_{2} \mathrm{O}+\mathrm{C}_{6} \mathrm{H}_{8} \mathrm{O}_{6}$ \\
\hline M41 & 53.66 & $\mathrm{C}_{39} \mathrm{H}_{51} \mathrm{O}_{21} \mathrm{P}$ & 921.2341 & -1.5 & {$[\mathrm{M}+\mathrm{Cl}]^{-}$} & Epimedin $\mathrm{C}+\mathrm{H}_{2}-\mathrm{H}_{2} \mathrm{O}+\mathrm{HPO}_{3}$ \\
\hline M42 & 53.67 & $\mathrm{C}_{37} \mathrm{H}_{47} \mathrm{~N}_{3} \mathrm{O}_{22} \mathrm{~S}$ & 916.2292 & -0.9 & {$[\mathrm{M}-\mathrm{H}]^{-}$} & Rutin $+\mathrm{H}_{2}+\mathrm{C}_{10} \mathrm{H}_{15} \mathrm{~N}_{3} \mathrm{O}_{6} \mathrm{~S}$ \\
\hline
\end{tabular}

$+\mathrm{H}_{2}$ : reduction; $+\mathrm{O}$ : oxidation; $+\mathrm{H}_{2} \mathrm{O}$ : hydration; $+\mathrm{HPO}_{3}$ : phosphorylation; $+\mathrm{C}_{6} \mathrm{H}_{8} \mathrm{O}_{6}$ : glucuronidation; $+\mathrm{C}_{5} \mathrm{H}_{7} \mathrm{NO}_{3} \mathrm{~S}$ : acetyl cysteine conjugation; $+\mathrm{C}_{10} \mathrm{H}_{15} \mathrm{~N}_{3} \mathrm{O}_{6} \mathrm{~S}$ : glutathione S-conjugation; $-\mathrm{H}_{2} \mathrm{O}$ : dehydration; -COO: decarboxylation.

was consistent with previous literature [28] and was finally identified as achyranthoside D. Peak 51 was identified clearly as ginsenoside Ro with a standard substance, and its mass fragmentation pattern is demonstrated in detail (Figure S4). In the negative ion mode, ginsenoside Ro gave $[\mathrm{M}-\mathrm{H}]^{-}$ion at $\mathrm{m} / z$ 955.4884, along with two major fragment ions at $\mathrm{m} / z$ 793.4236 [M-H-Glc $^{-}$and 631.2021[M-H-2Glc $]^{-}$in mass spectrometry under high-energy conditions. From the cleavage pathway of these triterpenoid saponins, they tend to lose the sugar group at the C28 position first under the action of high energy of $\mathrm{MS}^{\mathrm{E}}$. The possible reason for this phenomenon is that the ester bond at the $\mathrm{C} 28$ position is easier to break than the ether bond at the $\mathrm{C} 3$ position.
3.5.3. Phenylpropanoids. Six phenylpropanoids were recognized as the major active ingredients in GSL. Among them, a total of 2 phenylpropionic acids, 1 phenylpropanol, 1 styrene, and 2 lignans were identified. Simple phenylpropanoids belong to phenylpropane derivatives in structure and exist in plants in the form of glycosides or esters, which can be combined with sugars and polyols. In mass spectrometric analysis, phenylpropanoids are mainly manifested by the loss of sugar, neutral loss, and loss of other complex groups [29]. Compound 11 showed a deprotonated molecule $[\mathrm{M}-\mathrm{H}]^{-}$at $\mathrm{m} / z \quad 539.2149$ and produced predominant fragment ions at $\mathrm{m} / z 491.1857\left[\mathrm{M}-\mathrm{H}-\mathrm{CH}_{2} \mathrm{O}-\mathrm{H}_{2} \mathrm{O}\right]^{-}, \mathrm{m} / z$ $479.0789 \quad\left[\mathrm{M}-\mathrm{H}-\mathrm{C}_{2} \mathrm{H}_{4} \mathrm{O}_{2}\right]^{-}$, and $\mathrm{m} / z \quad 317.0238$ 


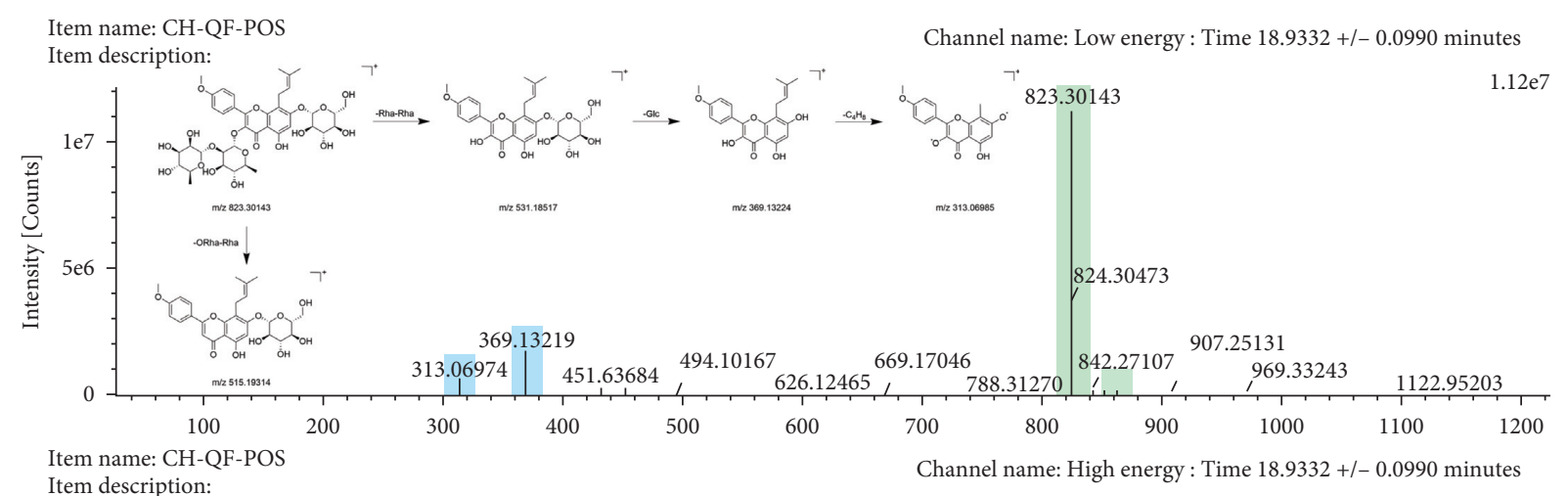

Item description:

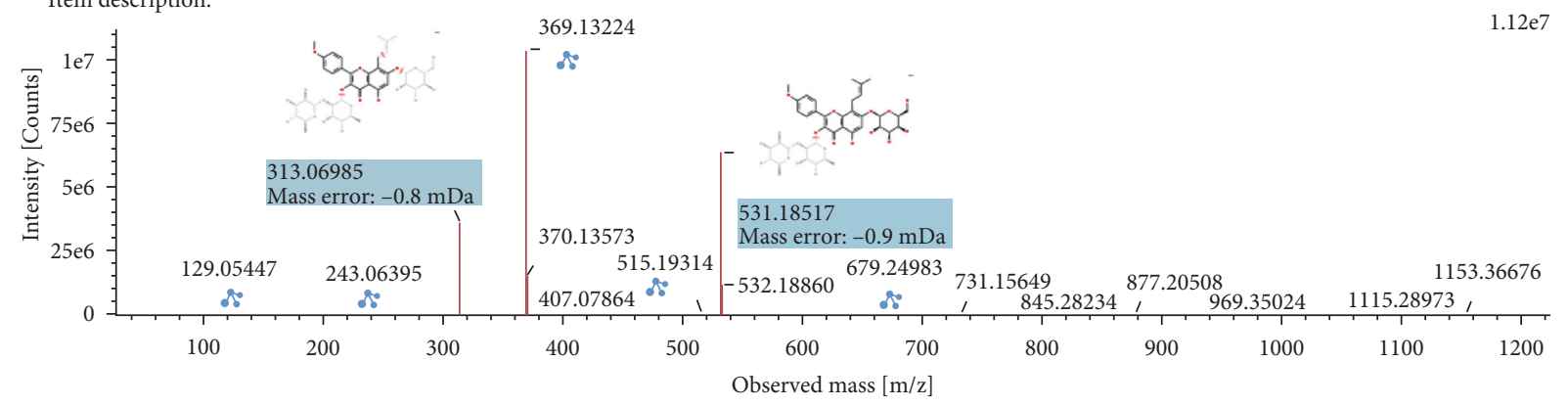

FIgURE 3: The mass spectrogram and fragmentation pathways of epimedin $\mathrm{C}$ in positive ion mode.

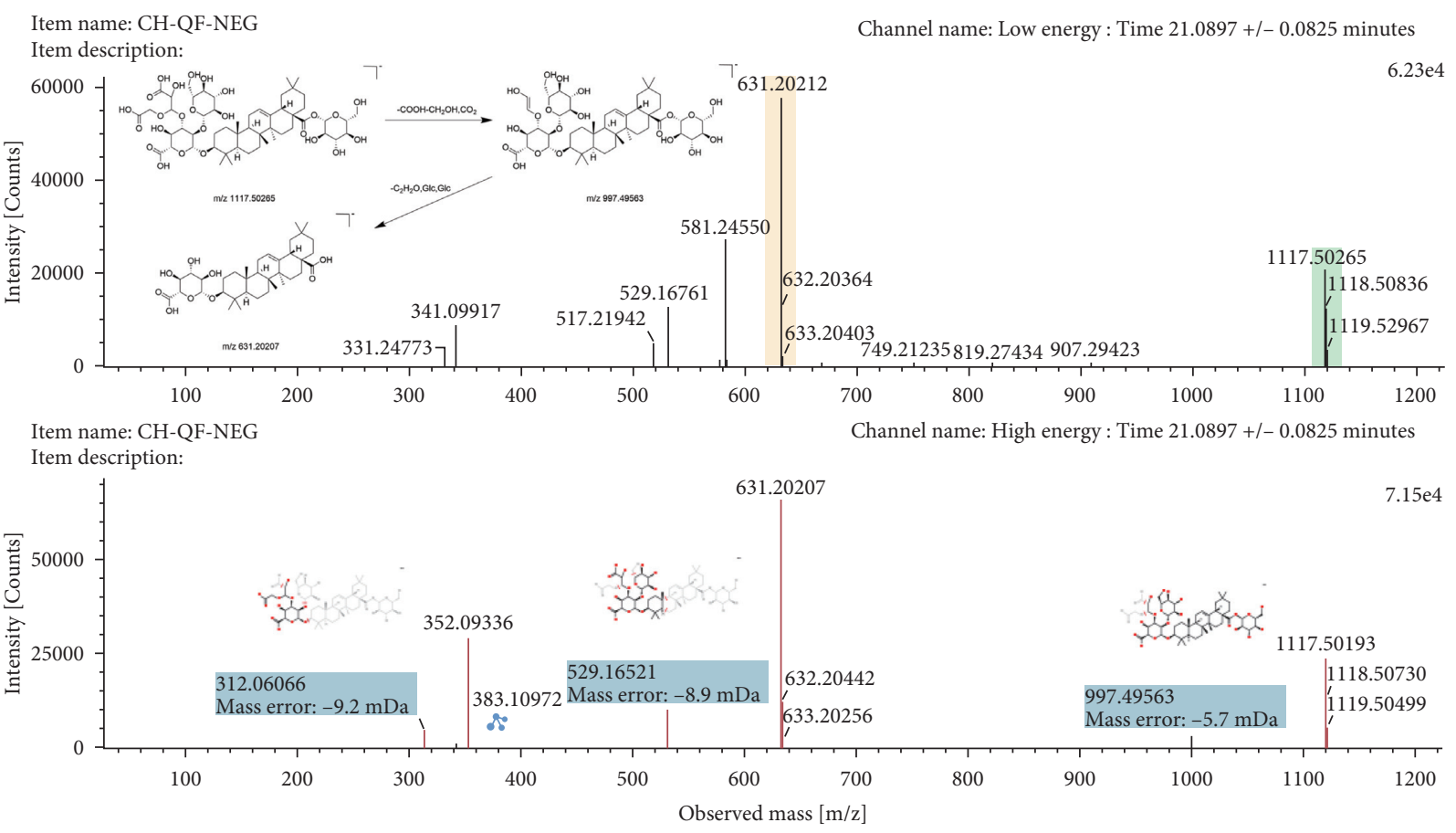

FIGURE 4: The mass spectrogram and fragmentation pathways of achyranthoside D in negative ion mode.

$\left[\mathrm{M}-\mathrm{H}-\mathrm{C}_{3} \mathrm{H}_{6} \mathrm{O}-\mathrm{Rha}-\mathrm{OH}\right]^{-}$in negative ion mode. By confirmation of fragment ions, we preliminarily identified compound 11 as icariside E1. In the case of compound 44, it generated a base peak ion at $\mathrm{m} / z 481.1785[\mathrm{M}+\mathrm{Na}]^{+}$in positive ion mode, along with a major fragment ion at $\mathrm{m} / z$ $387.1427\left[\mathrm{M}+\mathrm{Na}-\mathrm{C}_{4} \mathrm{H}_{8} \mathrm{O}\right]^{+}$, which was consistent with a previous study [30]. Finally, it was assigned to be rubschisantherin. The detailed mass spectrogram and fragmentation pathways are shown in Figures 5 and S5.

3.5.4. Steroids. In the UNIFI results interface, 5 steroids were automatically matched. The cleavage of steroids and their aglycones is complicated. Besides RDA cleavage, 


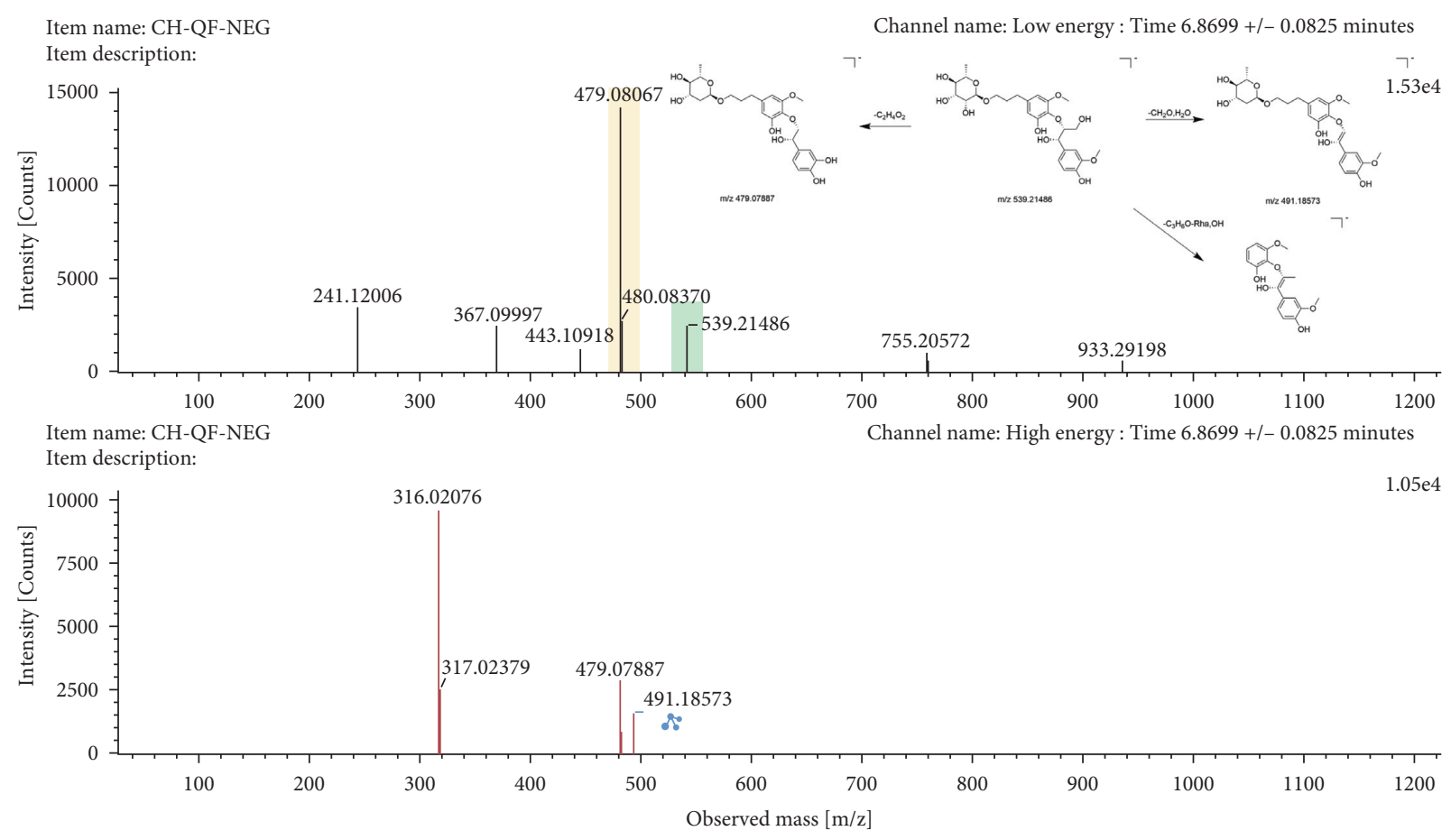

FIGURE 5: The mass spectrogram and fragmentation pathways of icariside E1 in negative ion mode.

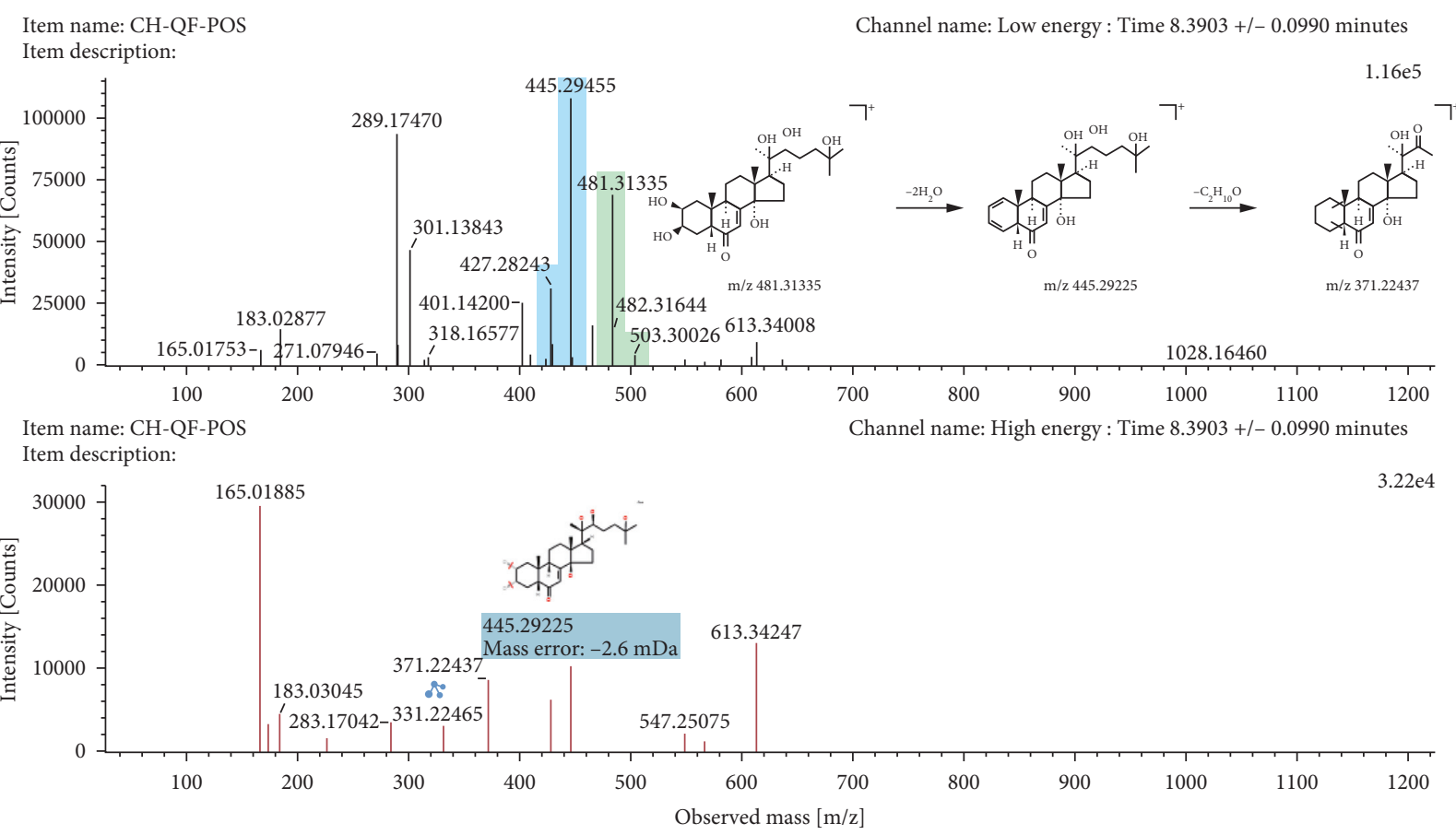

Figure 6: The mass spectrogram and fragmentation pathways of ecdysterone in positive ion mode.

dehydration, and demethylation of the hydroxyl group, the side chain at position 17 often falls off. Peak 18 was ascertained to be ecdysterone by contrast with reference standards. As shown in Figure 6, ecdysterone displayed a hydrogenated ion at $\mathrm{m} / z 481.3134[\mathrm{M}+\mathrm{H}]^{+}$with a molecular formula $\mathrm{C}_{27} \mathrm{H}_{44} \mathrm{O}_{7}$ and lost $\mathrm{H}_{2} \mathrm{O}$ to generate an ion at $\mathrm{m} / z$ 
445.2923 $\left[\mathrm{M}+\mathrm{H}-2 \mathrm{H}_{2} \mathrm{O}\right]^{+}$. Further loss of the $\mathrm{C}_{4} \mathrm{H}_{10} \mathrm{O}$ group resulted in fragmentation with a $\mathrm{m} / z$ of 371.2244 $\left[\mathrm{M}+\mathrm{H}-2 \mathrm{H}_{2} \mathrm{O}-\mathrm{C}_{4} \mathrm{H}_{10} \mathrm{O}\right]^{+}$.

3.5.5. Others. Some compounds with fewer species and lower concentrations are assigned to this category. The mass spectra data extracted from MassLynx workstation were matched with UNIFI software, and the results were verified by literature analysis [31]. A total of 13 compounds were inferred, including anthraquinones, glycosides, organic acids, and others. Specific mass spectrometry data are listed in Table 1.

\section{Conclusions}

In this experiment, UPLC-Q-TOF-MS technology combined with UNIFI software was used for the first time to comprehensively and systematically analyze the in vitro and in vivo chemical constituents of GSL. We summarized the cleavage law of flavonoids, triterpene saponins, phenylpropanoids, and steroids in the mass spectrum and initially explored the prototype compounds and metabolites of GSL in rat serum. These results provide a technical basis for the comprehensive and effective quality control and pharmacodynamic material basis of GSL. In addition, some chromatographic peaks with better response in GSL are unknown ingredients, which deserve further study.

\section{Data Availability}

The data used to support the findings of this study are included within the article.

\section{Conflicts of Interest}

The authors declare that there are no conflicts of interest regarding the publication of this paper.

\section{Acknowledgments}

This research was funded by the Project of Natural Science Research in Universities of Anhui Province (KJ2019A0314), National Natural Science Foundation of China (51303006 and 81703805), and the Provincial Natural Science Foundation of Anhui Province (KJ2021ZD0065 and 1408085MH196).

\section{Supplementary Materials}

See Table S1 and Figures S1-S5 in Supplementary Materials for comprehensive images and value analysis. (Supplementary Materials)

\section{References}

[1] M. Rossini, S. Adami, F. Bertoldo et al., "Guidelines for the diagnosis, prevention and management of osteoporosis," Reumatismo, vol. 68, no. 1, pp. 1-39, 2016.
[2] R. Nuti, M. L. Brandi, G. Checchia et al., "Guidelines for the management of osteoporosis and fragility fractures," Internal and Emergency Medicine, vol. 14, no. 1, pp. 85-102, 2019.

[3] F. S. O'Ryan and J. C. Lo, "Bisphosphonate-related osteonecrosis of the jaw in patients with oral bisphosphonate exposure: clinical course and outcomes," Journal of Oral and Maxillofacial Surgery, vol. 70, no. 8, pp. 1844-1853, 2012.

[4] T. D. Rachner, U. Platzbecker, D. Felsenberg, and L. C. Hofbauer, "Osteonecrosis of the jaw after osteoporosis therapy with denosumab following long-term bisphosphonate therapy," Mayo Clinic Proceedings, vol. 88, no. 4, pp. 418-419, 2013.

[5] J. E. Rossouw, G. L. Anderson, R. L. Prentice et al., "Risks and benefits of estrogen plus progestin in healthy postmenopausal women: principal results from the Women's Health Initiative randomized controlled trial," Journal of the American Medical Association, vol. 288, pp. 321-333, 2002.

[6] M. R. Meyer and M. Barton, "Estrogens and coronary artery disease," Advances in Pharmacology, vol. 77, pp. 307-360, 2016.

[7] T. Wang, Q. Liu, W. Tjhioe et al., "Therapeutic potential and outlook of alternative medicine for osteoporosis," Current Drug Targets, vol. 18, pp. 1051-1068, 2017.

[8] N.-D. Zhang, T. Han, B.-K. Huang et al., "Traditional Chinese medicine formulas for the treatment of osteoporosis: implication for antiosteoporotic drug discovery," Journal of Ethnopharmacology, vol. 189, pp. 61-80, 2016.

[9] L. Wang, Y. Li, Y. Guo et al., "Herba Epimedii: an ancient Chinese Herbal Medicine in the prevention and treatment of osteoporosis," Current Pharmaceutical Design, vol. 22, pp. 328-349, 2016.

[10] C. H. Fang, J. Liu, X. P. Zhang, M. X. Han, Y. Z. Zhang, and H. M. Nie, "Clinical observation on 60 cases of senile osteoporosis treated by Gushuling," Traditional Chinese Medicine and Clinical Pharmacology, vol. 14, pp. 30-32, 1999.

[11] D. Y. Wang and C. H. Fang, "Report of 60 cases of senile osteoporosis treated by Gushuling," Traditional Chinese Medicine and Clinical Pharmacology, vol. 20, pp. 55-56, 2008.

[12] T. Yin and C. H. Fang, "Clinical observation of Gushuling in preventing and treating postmenopausal osteoporosis," Anhui Medical and Pharmaceutical Journal.vol. 12, pp. 863-864, 2008.

[13] Y. Q. Shu, C. H. Fang, and T. T. Ba, "Clinical observation on treatment of diabetic osteoporosis by warming yang, nourishing qi and activating blood," Journal of Emergency in Traditional Chinese Medicine, vol. 19, pp. 49-50, 2010.

[14] J. S. Rooney, A. McDowell, C. J. Strachan, and K. C. Gordon, "Evaluation of vibrational spectroscopic methods to identify and quantify multiple adulterants in herbal medicines," Talanta, vol. 138, pp. 77-85, 2015.

[15] Y. Jiang, B. David, P. Tu, and Y. Barbin, "Recent analytical approaches in quality control of traditional Chinese medicines-a review," Analytica Chimica Acta, vol. 657, no. 1, pp. 9-18, 2010.

[16] J. Yu, R. Zhu, Y. J. Dong, Y. L. Zhu, R. Sang, and W. D. Chen, "Effects of the compatibility of Gushuling granules on the icariin content," Chinese Journal of Pharmaceuticals, vol. 27, pp. 79-82, 2016.

[17] R. Sang, X. X. Wang, Y. Y. Zhu, Y. L. Zhu, and W. D. Chen, "Serum pharmacochemistry analysis of Gushuling in rats," Traditional Chinese Medicine in the Treatment of Patients, vol. 21, pp. 78-81, 2015.

[18] G. Zhou, M. Wang, Y. Li, R. Xu, and X. Li, “Comprehensive analysis of 61 characteristic constituents from Siraitiae fructus 
using ultrahigh-pressure liquid chromatography with timeof-flight mass spectrometry," Journal of Pharmaceutical and Biomedical Analysis, vol. 125, pp. 1-14, 2016.

[19] M. Ganzera and S. Sturm, "Recent advances on HPLC/MS in medicinal plant analysis-An update covering 2011-2016," Journal of Pharmaceutical and Biomedical Analysis, vol. 147, pp. 211-233, 2018.

[20] H. Wu, Y. Chen, Q. Li et al., "Intervention effect of Qi-YuSan-Long decoction on lewis lung carcinoma in C57BL/6 mice: insights from UPLC-QTOF/MS-based metabolic profiling," Journal of Chromatography B, vol. 1102-1103, pp. 23-33, 2018.

[21] B. Zhu, H. Cao, L. Sun et al., "Metabolomics-based mechanisms exploration of Huang-Lian Jie-Du decoction on cerebral ischemia via UPLC-Q-TOF/MS analysis on rat serum," Journal of Ethnopharmacology, vol. 216, pp. 147-156, 2018.

[22] F.-X. Zhang, M. Li, L.-R. Qiao et al., "Rapid characterization of Ziziphi Spinosae Semen by UPLC/Qtof MS with novel informatics platform and its application in evaluation of two seeds from Ziziphus species," Journal of Pharmaceutical and Biomedical Analysis, vol. 122, pp. 59-80, 2016.

[23] L. Deng, A.-M. Shi, H.-Z. Liu et al., "Identification of chemical ingredients of peanut stems and leaves extracts using UPLCQTOF-MS coupled with novel informatics UNIFI platform," Journal of Mass Spectrometry, vol. 51, no. 12, pp. 1157-1167, 2016.

[24] L. Xu, Y. Liu, H. Wu, H. Wu, X. Liu, and A. Zhou, "Rapid identification of chemical profile in Gandou decoction by UPLC-Q-TOF-MSE coupled with novel informatics UNIFI platform," Journal of Pharmaceutical Analysis, vol. 10, no. 1, pp. 35-48, 2020.

[25] J. R. Gao, M. Q. Zhu, X. L. Wang, H. Wu, L. Shan, and Y. Wang, "Identification of chemical constituents in Huangdi Anxiao Capsules by UPLC-Q-TOF-MS ${ }^{\mathrm{E}}$ combined with UNIFI software," China Journal of Chinese Materia Medica, vol. 45, pp. 2395-2405, 2020.

[26] S. Wang, L. Chen, J. Leng, P. Chen, X. Fan, and Y. Cheng, "Fragment ion diagnostic strategies for the comprehensive identification of chemical profile of Gui-Zhi-Tang by integrating high-resolution MS, multiple-stage MS and UV information," Journal of Pharmaceutical and Biomedical Analysis, vol. 98, pp. 22-35, 2014.

[27] C.-J.-S. Lai, T. Tan, S.-L. Zeng et al., "An integrated high resolution mass spectrometric data acquisition method for rapid screening of saponins in Panax notoginseng (Sanqi)," Journal of Pharmaceutical and Biomedical Analysis, vol. 109, pp. 184-191, 2015.

[28] J. Fu, H. Wu, and $\mathrm{H}$. Wu, "Rapid characterization of triterpenoid saponins in Achyranthes bidentate Blume by UPLC-Q -TOF/MS in $\mathrm{MS}^{\mathrm{E}}$ mode combined with UNIFI screening platform," Natural Product Research and Development, vol. 31, pp. 1054-1061+1090, 2019.

[29] J. Xie, L. Zhang, J. X. Zeng et al., "Fast identification of constituents of Lagotis brevituba by using UPLC-Q-TOF-MS/ MS method," China Journal of Chinese Materia Medica, vol. 42, pp. 2123-2130, 2017.

[30] X.-M. Gao, R.-R. Wang, D.-Y. Niu et al., "Bioactive dibenzocyclooctadiene lignans from the stems of Schisandra neglecta," Journal of Natural Products, vol. 76, no. 6, pp. 1052-1057, 2013.

[31] D. Y. Lee, B.-R. Choi, J. W. Lee et al., "Simultaneous determination of various platycosides in Four Platycodon grandiflorum cultivars by UPLC-QTOF/MS," Applied Biological Chemistry, vol. 62, no. 1, p. 47, 2019. 